\title{
The Mathematics of Richard Schoen
}

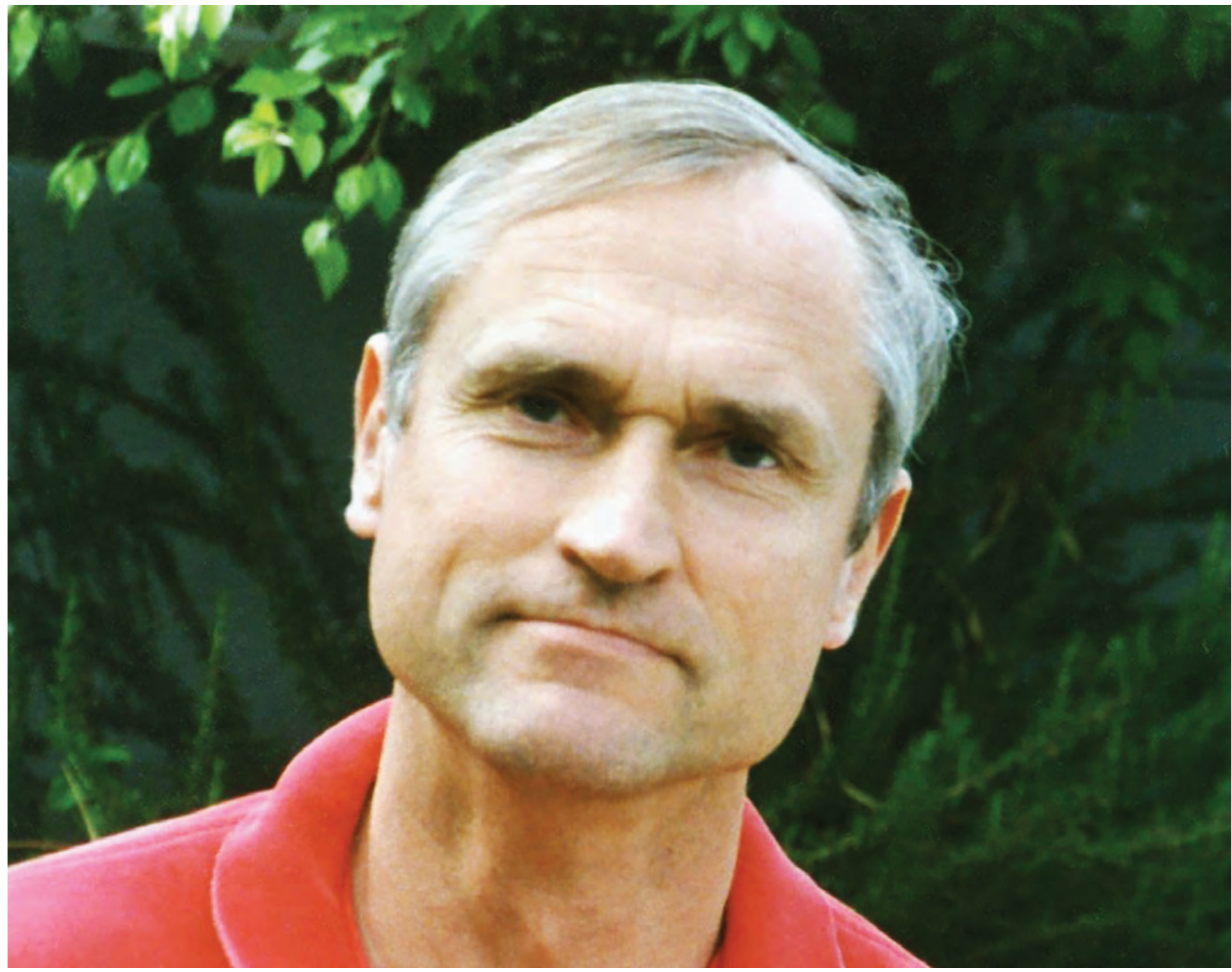

Communicated by Christina Sormani

For permission to reprint this article, please contact:

reprint-permission@ams.org.

DOI: http://dx.doi.org/10.1090/noti1749 


\section{Hubert L. Bray and William P. Minicozzi II}

\section{Preface}

For more than forty years Richard Schoen has been a leading figure in geometric analysis, connecting ideas between analysis, geometry, topology, and physics in fascinating and unexpected ways. In 2017 Richard Schoen was awarded the Wolf Prize for these fundamental contributions and for his "understanding of the interconnectedness of partial differential equations and differential geometry." In this article we survey some of his many fundamental ideas.
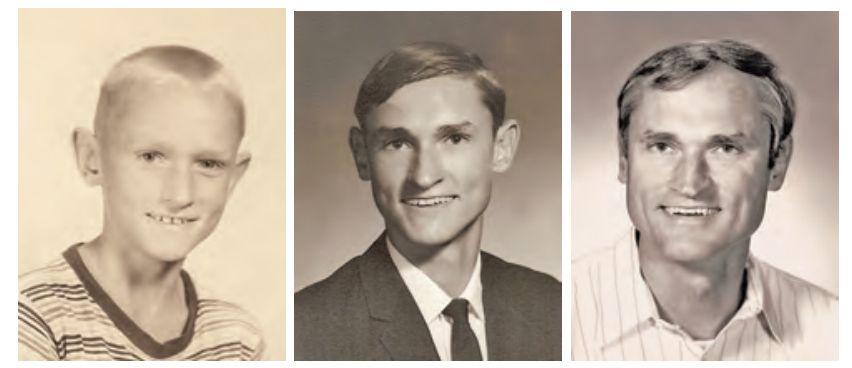

Figure 1. Schoen was the tenth of thirteen children, shown here in fourth grade at the Sharpsburg Elementary School, at graduation from Fort Recovery High School in 1968, and at graduation summa cum laude from the University of Dayton in 1972.

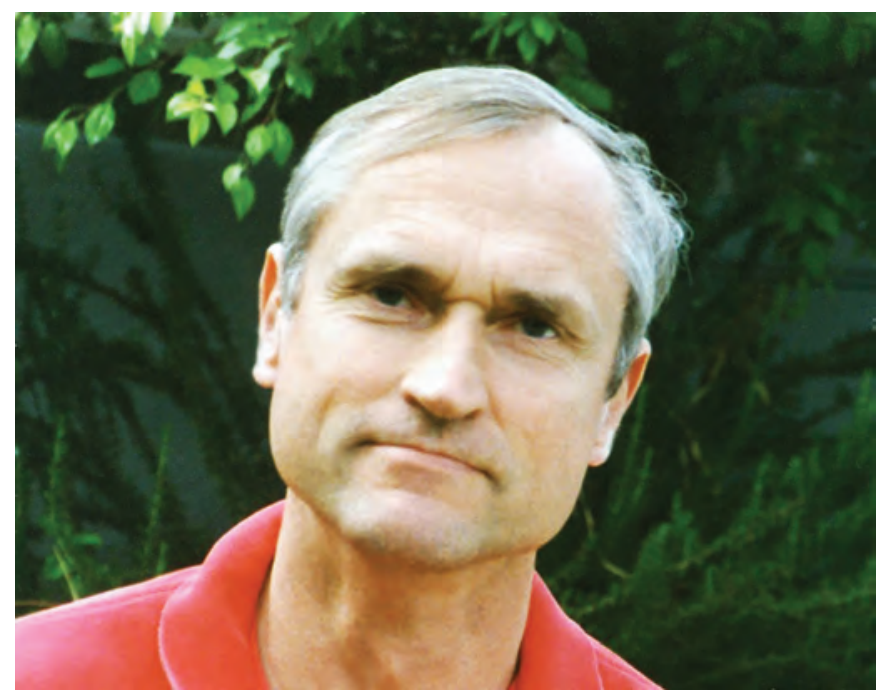

Figure 2. Rick Schoen, pictured here soon after winning the Guggenheim Fellowship in 1996, also won the Wolf Prize in 2017.

Rick Schoen was born in 1950 in Celina, Ohio. He was the tenth in a family of thirteen children growing

Hubert Bray is professor of mathematics at Duke University. His email address is hubert. bray@duke.edu.

William Minicozzi is Singer Professor of Mathematics at MIT. His email address is minicozz@math.mit.edu. up on a farm (Figure 1). He enjoyed farm work and has described driving a tractor to plow the fields as "great for thinking." His mother encouraged the children in their schooling, and his father was always inventing things. His older brothers, Hal and Jim, were both math majors and inspired him to study mathematics.

In 1972 Schoen (Figure 1) graduated summa cum laude from the University of Dayton and received an NSF Graduate Fellowship. In March 1977 Rick received his $\mathrm{PhD}$ from Stanford University under the direction of Leon Simon and Shing-Tung Yau and soon after received a Sloan Postdoctoral Fellowship. His early work was on minimal surfaces and harmonic maps. By the time Schoen received his $\mathrm{PhD}$, he had already proven major results, including his 1975 curvature estimates paper with Simon and Yau.

In the late 1970s Schoen and Yau developed new tools to study the topological implications of positive scalar curvature. This work grew out of their study of stable minimal surfaces, eventually leading to their proof of the positive mass theorem in 1979. Altogether, their work was impressive for the way it connected neighboring fields, first using analysis to understand geometry and then using geometry to understand physics.

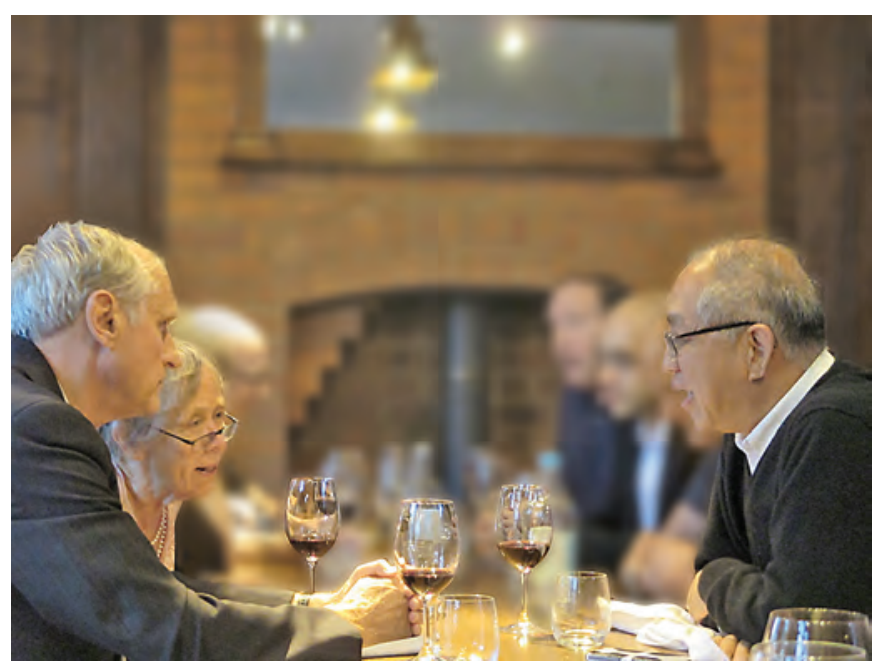

Figure 3. Schoen with his collaborators on the theory of stable minimal surfaces in manifolds of positive scalar curvature: Fischer-Colbrie and Fields Medalist Yau (2015). Yau and Schoen applied stable minimal surfaces to prove the positive mass theorem in 1979.

In the early 1980s, Schoen published a number of fundamental papers on minimal surfaces and harmonic maps. His work on minimal surfaces includes an influential Bernstein theorem for stable minimal surfaces with Doris Fischer-Colbrie. Schoen met his future wife, FischerColbrie, in Berkeley, where she received her PhD in 1978. They have two children, Alan and Lucy, both of whom graduated from Stanford.

Other works from the early 1980s include an extremely useful curvature estimate for stable surfaces, a uniqueness theorem for the catenoid, and a partial regularity 
theory for stable hypersurfaces in high dimensions with Simon. In 1982, Schoen and Karen Uhlenbeck proved the partial regularity of energy-minimizing harmonic maps. In 1983 Schoen was awarded the very prestigious MacArthur Prize Fellowship.

Schoen is also very well known for his celebrated solution to the remaining cases of the Yamabe problem in 1984, this time using a theorem from physics, namely the positive mass theorem, to solve a famous problem in geometry. The resulting fundamental theorem in geometry, that every smooth Riemannian metric on a closed manifold admits a conformal metric of constant scalar curvature, had been open since the 1960s. This work was cited in 1989 when Schoen received the Bocher Prize of the American Mathematical Society. His work on scalar curvature at this time set the direction for the field for the next twenty-five years.

Schoen was elected to the American Academy of Arts and Sciences in 1988 and the National Academy of Sciences in 1991. He has been a Fellow of the American Association for the Advancement of Science since 1995 and won a Guggenheim Fellowship in 1996. Rick was elected Vice President of the AMS in 2015. He was awarded the Wolf Prize in Mathematics for 2017, shared with Charles Fefferman. In 2017 he was also awarded the Heinz Hopf Prize, the Lobachevsky Medal and Prize, and the Rolf Schock Prize, to mention only a few of his awards.

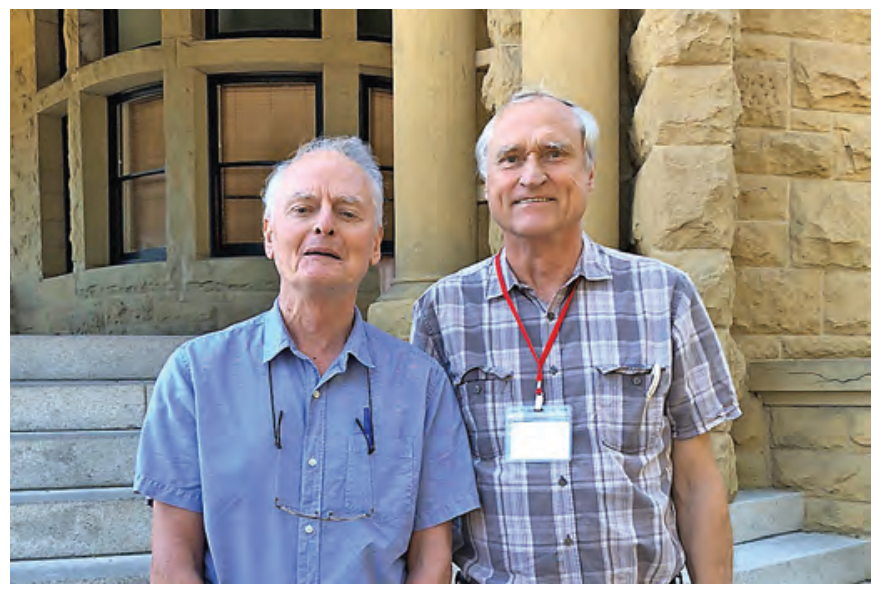

Figure 4. Schoen with Simon at Stanford in 2018. Schoen is Simon's most successful doctoral student, with 137 mathematical descendants.

Starting around 1990 Schoen began two major programs. The first was to develop a theory of harmonic maps with singular targets, starting with a joint paper with Mikhail Gromov, where they used harmonic maps to establish $p$-adic superrigidity for lattices in groups of rank one. In a series of papers, Schoen and Nick Korevaar laid the foundations for a general theory of mappings to NPC spaces, established the basic existence and regularity results, and applied their theory to settle problems in a number of areas of mathematics. The second big program was a variational theory of Lagrangian submanifolds, including the existence and regularity theory, done in a series of papers with Jon Wolfson.

Over the last decade, Schoen has continued to make major contributions to geometric analysis and general relativity. Among other results in general relativity, Schoen has made fundamental contributions to the constraint equations (with Corvino and others), which dictate the range of possible initial conditions for a spacetime, proved theorems on the topology of higher-dimensional black holes (with Galloway), and proved the positive mass theorem in dimensions greater than seven (with Yau). In geometric analysis he has several important results with Simon Brendle on Ricci flow, including the proof of the differentiable sphere theorem, as well as a compactness theorem for the Yamabe equation with Marcus Khuri and Fernando Codá Marques.

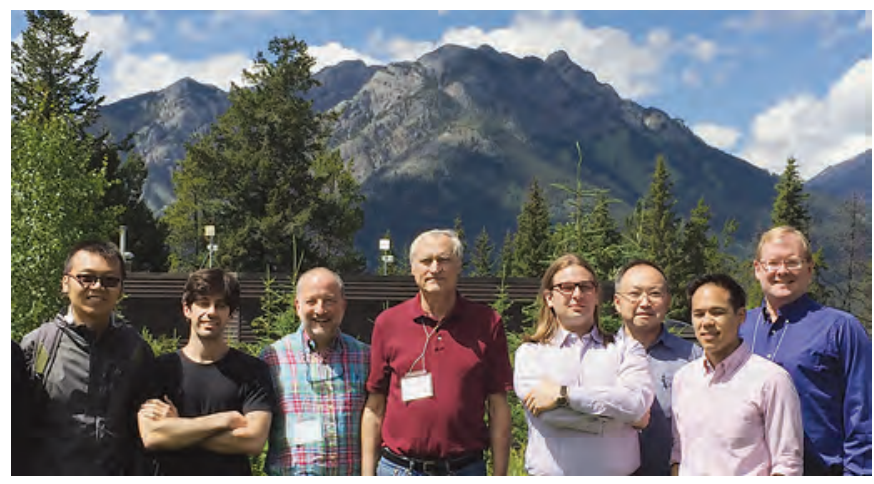

Figure 5. Schoen at the Banff International Research Station in 2016 with his students Pengzi Miao, Christos Mantoulidis, Daniel Pollack, Alessandro Carlotto, Sumio Yamada, Dan Lee, and Hubert Bray.

Schoen has written two books and roughly eighty papers and has solved an impressively wide variety of major problems and conjectures. He has supervised around forty students and counting and has hosted many postdocs. Even with his great success, Schoen is still one of the hardest working people in mathematics, giving us all the distinct impression that he must love it. His impact on mathematics, both in terms of his ideas and the example he sets, continues to be tremendous.

This article contains surveys of a sampling of Schoen's mathematical works, interspersed with personal recollections. Many of Schoen's accomplishments are not surveyed here-there were just too many to attempt that feat. Nevertheless, we hope that the reader will get a taste of the mathematical genius that is Richard Schoen.

In the next sections, Michael Eichmair and Lan-Hsuan Huang describe Schoen's work on scalar curvature, followed by a personal note by Shing-Tung Yau about their collaboration on the positive mass theorem. William Minicozzi describes the work of Schoen-Uhlenbeck, followed by a personal note by Karen Uhlenbeck about their collaboration. Rob Kusner describes Schoen's work on classical minimal surfaces in Euclidean space, and Fernando Codá Marques describes his work on the Yamabe problem. 


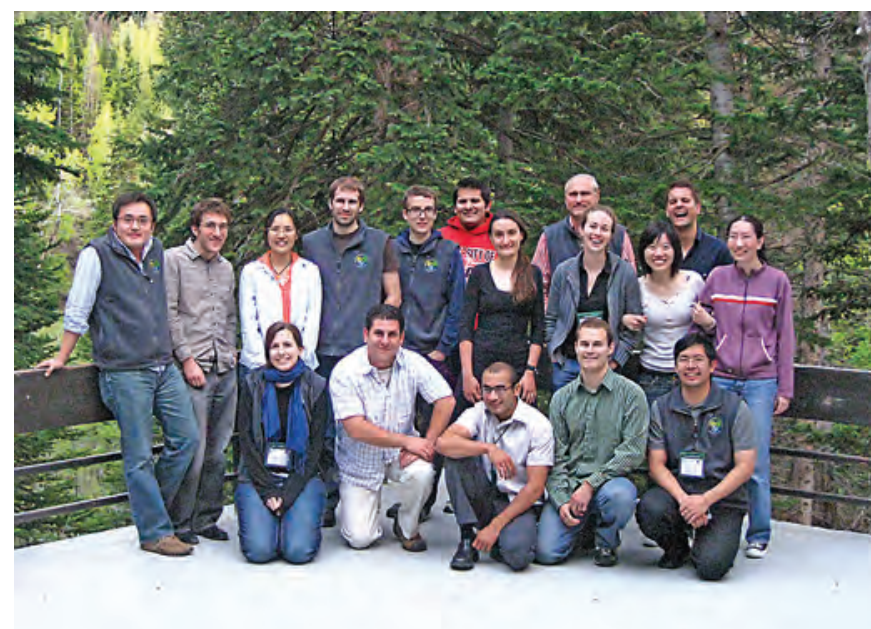

Figure 6. At the June 2009 Mathematics Research Communities Conference in Snowbird, Utah. First row: Catherine Williams, Vincent Bonini, Leobardo Rosales, Jeff Jauregui, Po-Ning Chen. Second row: George Lam, Jacob Bernstein, Chen-Yun Lin, Andrew Bulawa, Graham Cox, Joel Kramer, Iva Stavrov, Rick Schoen, Christine Breiner, Lan-Hsuan Huang, Michael Eichmair, Lu Wang.

Chikako Mese describes his work on harmonic maps into NPC spaces, and Ailana Fraser describes their joint work on Steklov eigenvalues. Any of these sections may be read as its own distinct contribution.

\section{Michael Eichmair and Lan-Hsuan Huang}

\section{Scalar Curvature, Minimal Surfaces, and the Positive Mass Theorem}

Here we describe some of Richard Schoen's early work with Shing-Tung Yau on the use of minimal surfaces in the study of three-dimensional geometry. We also describe the impact of their contributions on our own careers.

To set the stage, we briefly recall several notions of curvature. Let $(M, g)$ be a Riemannian manifold of dimension $n$.

Let $\pi$ be a 2-plane in the tangent space of $M$ at $p$. The sectional curvature of $\pi$ may be computed from the spread of geodesics $\gamma_{i}$ starting at $p$ with orthonormal initial velocities $e_{i}$ such that $\pi=e_{1} \wedge e_{2}$ :

$$
K_{p}(\pi)=\lim _{t \rightarrow 0} 6\left(\frac{t-\operatorname{dist}\left(\gamma_{i}(t), \gamma_{j}(t)\right) / \sqrt{2}}{t^{3}}\right) .
$$

The sectional curvature is positive when these geodesics bend together and negative when they drift apart when

Michael Eichmair is professor of mathematics at the University of Vienna. His email address is mi chae 1 . ei chmai r@uni vie . ac . at. Lan-Hsuan Huang is associate professor of mathematics at the University of Connecticut. Her email address is 1an-hsuan .huang@uconn.edu. compared with Euclidean space. Starting with an orthonormal basis $e_{1}, \ldots, e_{n}$ of the tangent space at $p$, we can compute the (symmetric) Ricci curvature tensor as

$$
\operatorname{Ric}_{p}\left(e_{i}, e_{i}\right)=\sum_{j \neq i} K_{p}\left(e_{i} \wedge e_{j}\right)
$$

and the scalar curvature as

$$
R_{p}=\sum_{i=1}^{n} \operatorname{Ric}_{p}\left(e_{i}, e_{i}\right)=\sum_{i \neq j} K_{p}\left(e_{i} \wedge e_{j}\right) .
$$

The scalar curvature can also be computed from the deficit between the volume $\omega_{n} r^{n}$ of a Euclidean ball of radius $r>0$ and the volume of a geodesic ball $B_{p}(r)=\{x \in M: \operatorname{dist}(x, p)<r\}$ in $(M, g):$

$$
R_{p}=\lim _{r \rightarrow 0} 6(n+2)\left(\frac{\omega_{n} r^{n}-\operatorname{vol}\left(B_{p}(r)\right)}{\omega_{n} r^{n+2}}\right) .
$$

Thus $R_{p}>0$ means that a small geodesic ball in $(M, g)$ has less volume than a Euclidean ball of the same radius.

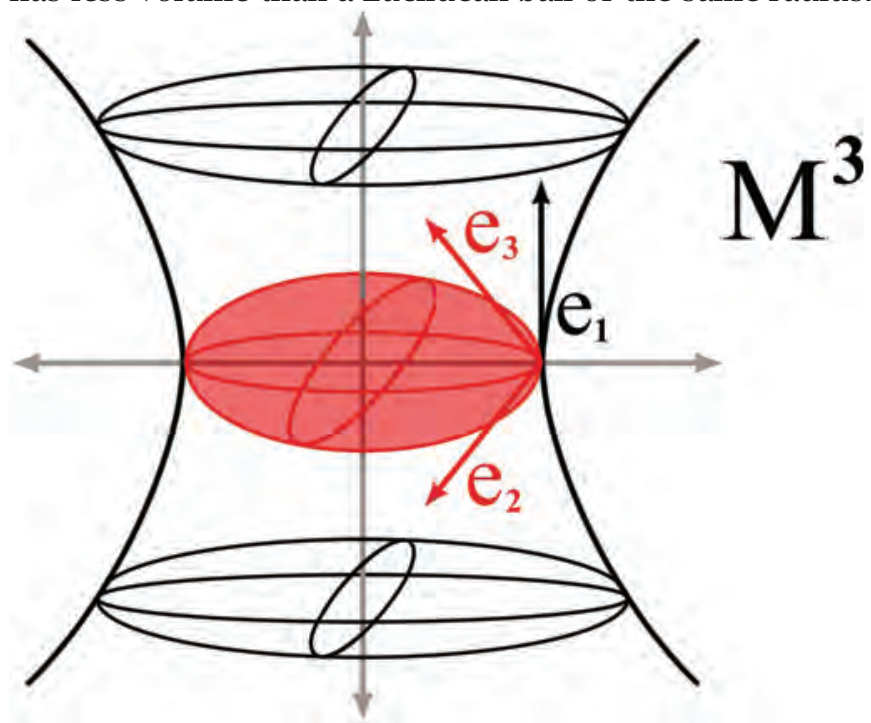

Figure 7. The spatial Schwarzschild geometry, with vanishing scalar curvature, provides the prototype for the concept of mass in general relativity. The sphere at the neck (in red) is a stable minimal surface called the horizon.

Let $\Sigma \subset M$ be a hypersurface. The variation $\nabla_{X} v$ of the unit normal $v: \Sigma \rightarrow T M$ along a field $X$ that is tangent to $\Sigma$ measures how $\Sigma$ bends in ambient space. These variations are recorded synthetically in a symmetric tensor on $\Sigma$,

$$
A_{\Sigma}(X, Y)=g\left(\nabla_{X} v, Y\right),
$$

called the second fundamental form. The principal curvatures $\lambda_{1}, \ldots, \lambda_{n-1}$ of $\Sigma$ at a given point are the eigenvalues of this tensor with respect to the inner product induced on $\Sigma$ by $g$. Their sum

$$
H_{\Sigma}=\lambda_{1}+\cdots+\lambda_{n-1}
$$

is called the mean curvature $H_{\Sigma}$ of $\Sigma$.

We examine mean curvature more carefully. Let us assume, for definiteness, that $\Sigma$ is closed. Every smooth 
function, $f \in C^{\infty}(\Sigma)$, with sufficiently small $C^{1}$-norm gives rise to a surface

$$
\Sigma_{f}=\left\{\exp _{\sigma} f(\sigma) \nu(\sigma): \sigma \in \Sigma\right\}
$$

near $\Sigma$. In fact, every surface close to $\Sigma$ has this form. Taylor expansion gives

$$
\begin{gathered}
\operatorname{area}\left(\Sigma_{f}\right)=\operatorname{area}(\Sigma)+\int_{\Sigma} H_{\Sigma} f+\frac{1}{2} \int_{\Sigma} H_{\Sigma}^{2} f^{2} \\
+\frac{1}{2} \int_{\Sigma}\left|\nabla_{\Sigma} f\right|^{2}-\left(\left|A_{\Sigma}\right|^{2}+\operatorname{Ric}(\nu, v)\right) f^{2} \\
+O\left(\|f\|_{C^{1}(\Sigma)}^{3}\right) .
\end{gathered}
$$

Assume now that $\Sigma$ has least area among all nearby surfaces. In particular, the first variation of area is zero. By the preceding formula, this is equivalent to

$$
H_{\Sigma}=0 \text {. }
$$

Moreover, the second variation of area is nonnegative. In view of our expansion, this amounts to the stability inequality, i.e.,

$$
\int_{\Sigma}\left(\left|A_{\Sigma}\right|^{2}+\operatorname{Ric}(v, v)\right) f^{2} \leq \int_{\Sigma}\left|\nabla_{\Sigma} f\right|^{2}
$$

for all $f \in C^{\infty}(\Sigma)$.

In general, we call a surface minimal if its mean curvature vanishes. Stable minimal surfaces are those that satisfy both the first and the second derivative tests for least area.

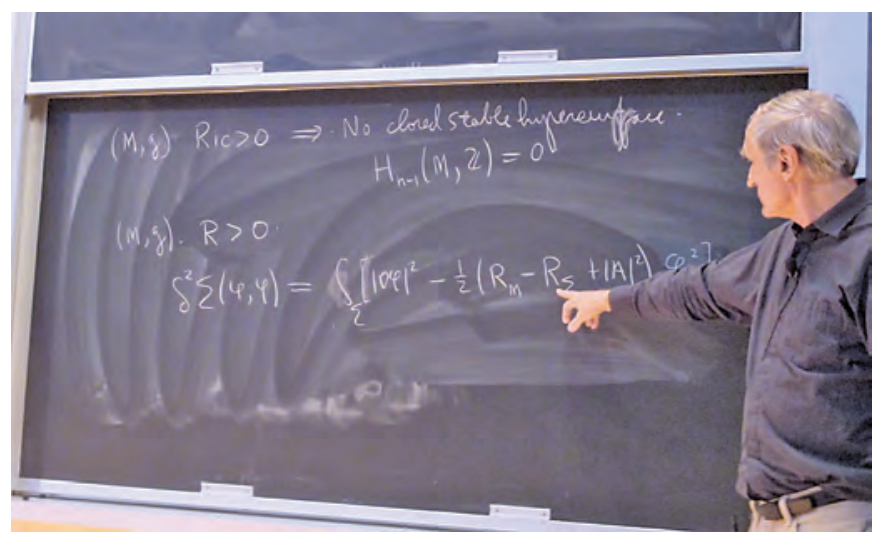

Figure 8. Schoen and Yau's famous manipulation of the second variation formula for a stable minimal surface, $\Sigma$, to involve the ambient scalar curvature, $R_{M}$, the intrinsic Gauss curvature, $R_{\Sigma}$, and the norm of the second fundamental form squared, $|A|^{2}$.

Using parallel surfaces for area comparison, where $f \equiv$ constant, is tempting and hard to resist. It gives

$$
\int_{\Sigma}\left|A_{\Sigma}\right|^{2}+\operatorname{Ric}(v, v) \leq 0 .
$$

J. Simons observed from this that there can be no stable minimal surfaces if the Ricci curvature is positive. Schoen and Yau [4] manipulated the integrand further, using the Gauss equation

$$
R=2 K_{\Sigma}+\left|A_{\Sigma}\right|^{2}-H_{\Sigma}^{2}+2 \operatorname{Ric}(v, v)
$$

and the Gauss-Bonnet formula

$$
\int_{\Sigma} K_{\Sigma}=2 \pi x(\Sigma)
$$

to conclude that, for a stable minimal surface,

$$
\frac{1}{2} \int_{\Sigma} R+\left|A_{\Sigma}\right|^{2} \leq \int_{\Sigma} K_{\Sigma}=2 \pi \chi(\Sigma) .
$$

Inspecting this identity, Schoen and Yau observed that the following two conditions-one metric and one topological-cannot hold simultaneously:

- The scalar curvature $R$ is positive along $\Sigma$.

- The genus of $\Sigma$ is positive; i.e., $\chi(\Sigma) \leq 0$.

Consider $(M, g)$ where $M=\mathbb{S}^{1} \times \mathbb{S}^{1} \times \mathbb{S}^{1}$ is the 3 torus. From standard results in geometric measure theory, among all surfaces in $M$ that are homologous to $\Sigma_{0}=$ $\mathbb{S}^{1} \times \mathbb{S}^{1} \times\{$ point $\}$, there is one that has least area in $(M, g)$; see Figure 9. By Stokes' theorem, this surface has a component $\Sigma$ for which

$$
\int_{\Sigma}\left(d \theta^{1}\right) \wedge\left(d \theta^{2}\right) \neq 0
$$

where $\theta^{1}, \theta^{2}$ are the angles on the first two factors. Assume that $\Sigma$ has genus zero. The restrictions of the forms $d \theta^{1}$ and $d \theta^{2}$ and consequently of $d \theta^{1} \wedge d \theta^{2}$ are exact on $\Sigma$. In particular, the latter should integrate to zero on $\Sigma$. This contradiction shows that $\Sigma$ has positive genus. In view of the dichotomy discussed above, these ideas of Schoen and Yau give

Theorem 1 (Schoen and Yau [3]). The 3-torus does not admit a metric of positive scalar curvature.

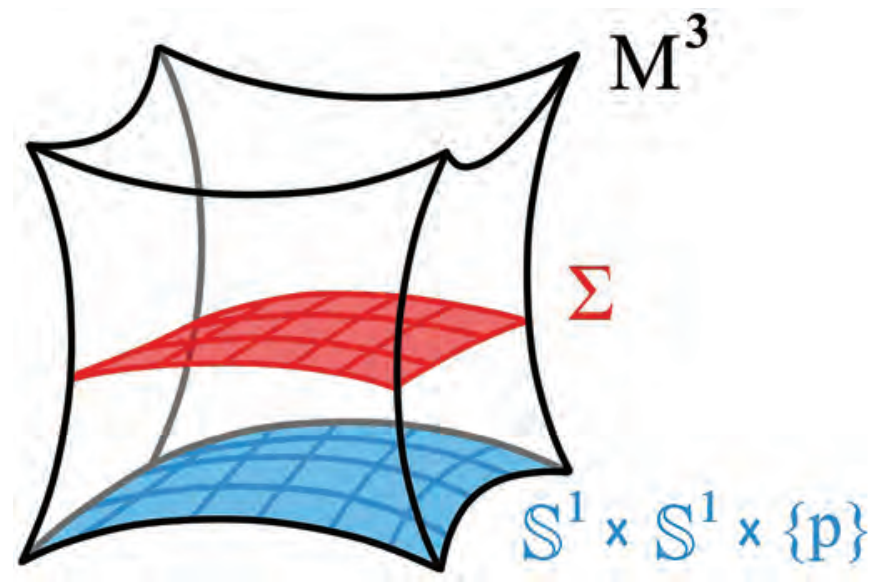

Figure 9. There is a stable minimal surface $\Sigma$ (in red) lying in a torus $M$ (in black), which is homologous to $\Sigma_{0}$ (in blue).

As shown by Kazdan-Warner in 1975, a Riemannian metric with nonnegative scalar curvature is either Ricci flat or there is a metric with positive scalar curvature nearby. Theorem 1 is thus really a rigidity result for Riemannian metrics of nonnegative scalar curvature. That is, every 3 -dimensional torus with nonnegative scalar curvature is flat.

Schoen and Yau extended these ideas in their proof of the positive mass theorem: 
Theorem 2 (Schoen and Yau [4]). Let $(M, g)$ be a complete Riemannian 3-manifold that is asymptotically flat,

$$
g_{i j}=\delta_{i j}+O\left(|x|^{-q}\right),
$$

for some $q>1 / 2$, with nonnegative integrable scalar curvature. Then the ADM-mass of $(M, g)$ is nonnegative, and it is zero if and only if $(M, g)$ is flat Euclidean space.

The ADM-mass (after R. Arnowitt, S. Deser, and C. Misner),

$$
m_{A D M}=\lim _{r \rightarrow \infty} \frac{1}{16 \pi r} \int_{\{|x|=r\}} \sum_{i, j=1}^{3}\left(\partial_{i} g_{i j}-\partial_{j} g_{i i}\right) x^{j},
$$

is a geometric invariant of $(M, g)$ that measures the deviation from Euclidean at infinity. The example of Schwarzschild where $M=\left\{x \in \mathbb{R}^{3}:|x| \geq m / 2\right\}$ and

$$
g_{i j}=u^{4} \delta_{i j} \quad \text { with } u(x)=1+\frac{m}{2|x|}
$$

for some $m>0$ is particularly important. The boundary of $M$ is a stable minimal surface and is called the horizon. Note that the ADM-mass is equal to $m$.

Schoen and Yau first consider the special case of harmonic asymptotics where, outside a bounded set,

$$
g_{i j}=u^{4} \delta_{i j} \quad \text { with } u(x)=1+\frac{m}{2|x|}+O\left(|x|^{-2}\right)
$$

and where the scalar curvature is positive everywhere. (The reduction of the proof of the positive mass theorem to the special case of such harmonic asymptotics is proven in a 1981 paper of Schoen and Yau using a density argument.) If $m=m_{A D M}$ is negative, the slab

$$
\left\{-\Lambda<x^{3}<\Lambda\right\}
$$

is a mean-convex region for $\Lambda>0$ sufficiently large. See Figure 10.

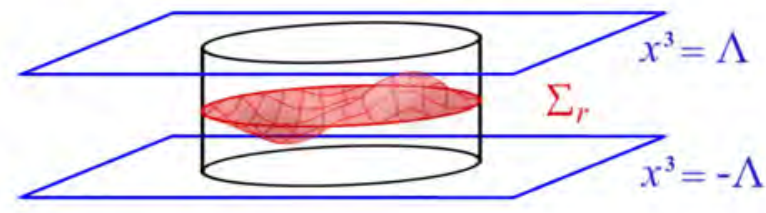

Figure 10. In their proof of the positive mass theorem, Schoen and Yau find a least area surface, $\Sigma_{r}$, with boundary, $\partial \Sigma_{r}$, lying in a cylinder of radius $r$, before taking $r \rightarrow \infty$ to create a complete stable minimal surface.

Schoen and Yau go ahead and construct least area surfaces in the slab with respective boundary

$$
\partial \Sigma_{r}=\left\{\left(x^{1}, x^{2}, 0\right):\left|\left(x^{1}, x^{2}\right)\right|=r\right\} .
$$

As $r \rightarrow \infty$, these surfaces limit to a complete stable minimal surface $\Sigma$ that is asymptotic to a horizontal plane. Since $\Sigma$ is unbounded, one cannot simply choose the constant test function 1 in the second variation inequality as before. Schoen and Yau apply an approximation argument involving a logarithmic cut-off trick to argue that, still,

$$
\int_{\Sigma}\left|A_{\Sigma}\right|^{2}+\operatorname{Ric}(v, v) \leq 0 .
$$

Using the Gauss equation as before, this gives

$$
0<\frac{1}{2} \int_{\Sigma} R \leq \int_{\Sigma} K_{\Sigma}
$$

The Cohn-Vossen inequality bounds the right-hand side by $2 \pi \chi(\Sigma)$. Now, using that $\Sigma$ is asymptotic to a plane, they conclude that $\chi(\Sigma) \leq 0$, a contradiction.

This shows that $m_{A D M} \geq 0$. To characterize the case of equality, Schoen and Yau use a perturbation argument.

These ideas of Schoen and Yau also extend to higher dimension.

Theorem 3 (Schoen and Yau [3]). Let $M$ be a closed manifold of dimension $n \leq 7$ so there exists a map $M \rightarrow \mathbb{T}^{n}=$ $\mathbb{S}^{1} \times \cdots \times \mathbb{S}^{1}$ of nonzero degree. Then $M$ does not admit a metric of positive scalar curvature.

The proof is by induction on the dimension. We may assume that $n \geq 4$. Using area-minimization, the stability inequality, and the Gauss equation as above, Schoen and Yau show that there is a hypersurface $\Sigma \subset M$ that admits a map of nonzero degree into the torus, $\mathbb{T}^{n-1}$, such that

$$
\frac{1}{2} \int_{\Sigma}\left(R+\left|A_{\Sigma}\right|^{2}\right) f^{2} \leq \int_{\Sigma}\left|\nabla_{\Sigma} f\right|^{2}+\frac{1}{2} R_{\Sigma} f^{2}
$$

for all $f \in C^{\infty}(\Sigma)$. Using the assumption that $R>0$, they conclude that the Yamabe operator

$$
L_{\Sigma}=-\Delta_{\Sigma}+\frac{n-3}{4(n-2)} R_{\Sigma}
$$

has positive first eigenvalue on $\Sigma$. If $u \in C^{\infty}(\Sigma)$ is a positive first eigenfunction, then the scalar curvature of the conformal metric $u^{\frac{4}{n-3}} g_{\Sigma}$ on $\Sigma$ is positive. This contradicts the induction hypothesis.

The restriction to low dimensions is on account of singularities that area-minimizing hypersurfaces may form. In recent work, Schoen and Yau present a powerful approach that extends their ideas to all dimensions.

Schoen and Yau have introduced a watershed of ideas relating scalar curvature, minimal surfaces, topology, and physics. Their insights have been hugely influential, and certainly a short survey cannot do them justice. We conclude by sketching briefly a few examples of how their ideas have influenced our own work.

The positive mass theorem is a remarkable rigidity result for Euclidean space: There really is no way at all to deform the Euclidean metric on a compact set while keeping the scalar curvature nonnegative. The following result due to Otis Chodosh and Eichmair establishes a related rigidity result that was conjectured by Schoen:

Theorem 4 ([1]). The only asymptotically flat 3-manifold that has nonnegative scalar curvature and which contains an unbounded area-minimizing surface is flat Euclidean space. 


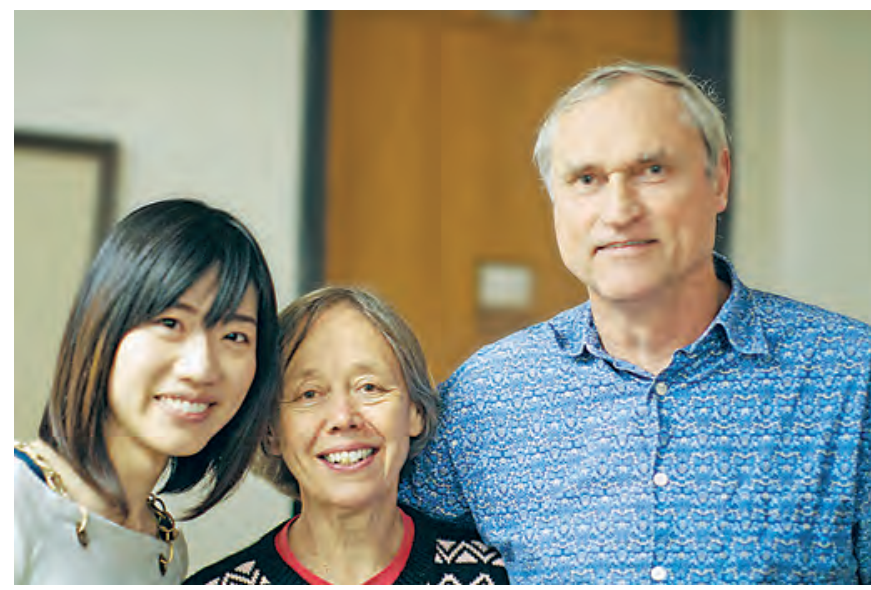

Figure 11. Huang, Fischer-Colbrie, and Schoen at Stanford (2007). Fischer-Colbrie and Schoen's paper "On the structure of complete stable minimal surfaces in 3-manifolds of positive scalar curvature" is one of Schoen's five most highly cited papers.

This result has already been used in the solution of several other conjectures related to the geometry of 3manifolds in joint work of Chodosh and Eichmair with Alessandro Carlotto, with Vlad Moraru, and with Yuguang Shi and Haobin Yu.

The reduction in the proof of the positive mass theorem to harmonic asymptotics described above illustrates how subtle results about the geometry of asymptotically flat manifolds can be obtained by looking at special cases.

This observation has found many useful applications. Huang studied further properties of the special classes that play a key role in studying the geometric center of mass, building on the foundational work for special data by Gerhard Huisken and Yau. This work was completed in her 2009 Stanford dissertation under Schoen's supervision. Schoen, Mu-Tao Wang, and Huang applied these ideas to study the angular momentum and disproved a conjectural mass and angular momentum inequality.

From the point of view of general relativity, asymptotically flat manifolds are just the beginning of the story. General initial data for the Einstein equations consists of triplets $(M, g, h)$, where $(M, g)$ is a Riemannian manifold and $h$ is a symmetric $(0,2)$-tensor that represents the spacetime second fundamental form of the slice $(M, g)$ through the evolving spacetime. ${ }^{1}$

In joint work with Dan A. Lee and Schoen [2], the authors proved a spacetime version of the positive mass theorem for initial data sets in dimensions $\leq 7$. The strategy is modeled on that for the time-symmetric case given by Schoen and Yau [5]. In our case, marginally outer trapped surfaces (MOTS) take the place of minimal surfaces. Since MOTS do not arise as minimizers of a geometric variational problem, we depend on existence,

${ }^{1}$ See the feature on gravitational waves in the August 2017 Notices, www. ams .org/publications/journals/notices/201707 /rnoti-p684.pdf. regularity, and compactness results for two-sided surfaces $\Sigma \subset M$ that solve a geometric boundary value problem of the form

$$
H_{\Sigma}(x)=F\left(x, v_{\Sigma}(x)\right)
$$

for all $x \in \Sigma$ with $\partial \Sigma=\Gamma$. Here, $F: M \times T M \rightarrow \mathbb{R}$ smooth and $\Gamma \subset M$ closed of codimension two are given. A theory that satisfyingly extends the classical theory for area-minimizing surfaces to this broader, nonvariational setting is developed in Eichmair's thesis under Schoen at Stanford in 2008. The accompanying rigidity result for Minkowski spacetime was obtained by Lee and Huang.

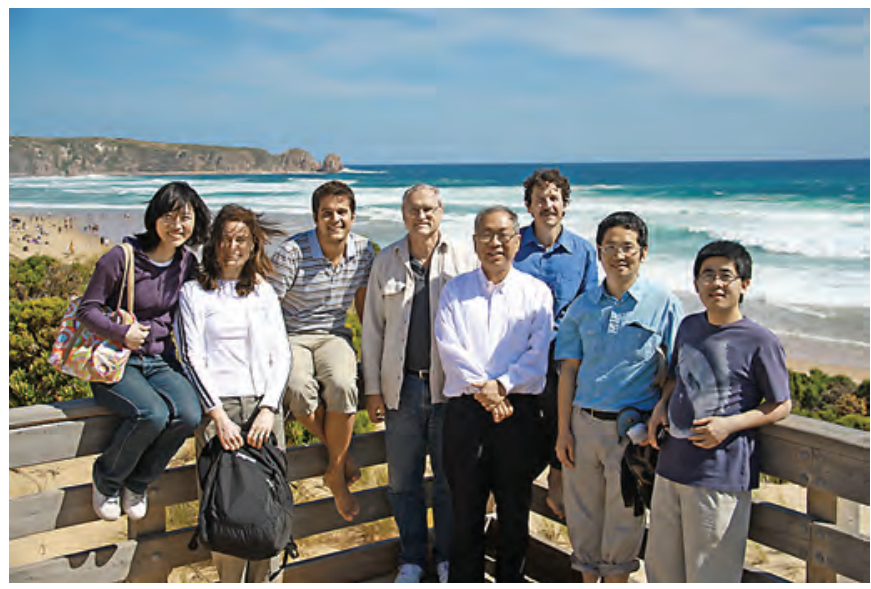

Figure 12. Lan-Hsuan Huang, Ailana Fraser, Michael Eichmair, Rick Schoen, Shing-Tung Yau, Robert Bartnik, Mu-Tao Wang, and Xiaodong Wang in Melbourne (2010).

On a personal note, we have very fond memories of our time working as doctoral students with Rick at Stanford2003-2008 (Eichmair) and 2004-2009 (Huang). He would be in the office seven days a week, typically from quite early in the morning until around $4 \mathrm{pm}$. (There was an ongoing search for tennis partners in the department for the late afternoons.) This example has rubbed off on his $\mathrm{PhD}$ students too. We worked hard in a nurturing environment that could not have been more down-toearth about mathematics. We aspired to be like Rick. Those years laid the foundations for our own research careers, and we are very grateful.

\section{References}

[1] A. CARlotto, O. Chodosh, and M. Eichmair, Effective versions of the positive mass theorem, Invent. Math. 206 (2016), 975-1016. MR3573977

[2] M. Eichmair, L.-H. HuAng, D. A. Lee, and R. Schoen, The spacetime positive mass theorem in dimensions less than eight, J. Eur. Math. Soc. 18 (2016), 83-121. MR3438380

[3] R. SCHOEN and S.-T. YAU, On the structure of manifolds with positive scalar curvature, Manuscripta Math. 28 (1979), 159183. MR0535700

[4] R. SCHOEN and S.-T. YAU, On the proof of the positive mass conjecture in general relativity, Comm. Math. Phys. 65 (1979), 45-76. MR0526976 
[5] R. Schoen and S.-T. YAU, Complete manifolds with nonnegative scalar curvature and the positive action conjecture in general relativity, Proc. Nat. Acad. Sci. U.S.A. 76 (1979), 1024-1025. MR0524327

\section{Shing-Tung Yau}

\section{Memories of Working with Rick Schoen}

I am pleased to write on behalf of Rick Schoen on the occasion of his Wolf Prize, which is clearly a welldeserved award in recognition of the tremendous insight and strength he has long demonstrated in the field of geometric analysis.

I have known Rick for forty-four years, and he is certainly one of my very best friends. I am proud that Leon Simon and I were his thesis advisors at Stanford. Together, Schoen, Leon Simon, Karen Uhlenbeck, S. Y. Cheng, Richard Hamilton, Clifford Taubes, and I made great strides in the 1970s in developing the modern theory of geometric analysis. This was a truly exciting period for the subject of differential geometry, laying the foundation for major developments in topology, algebraic geometry, and mathematical physics over the last four decades.

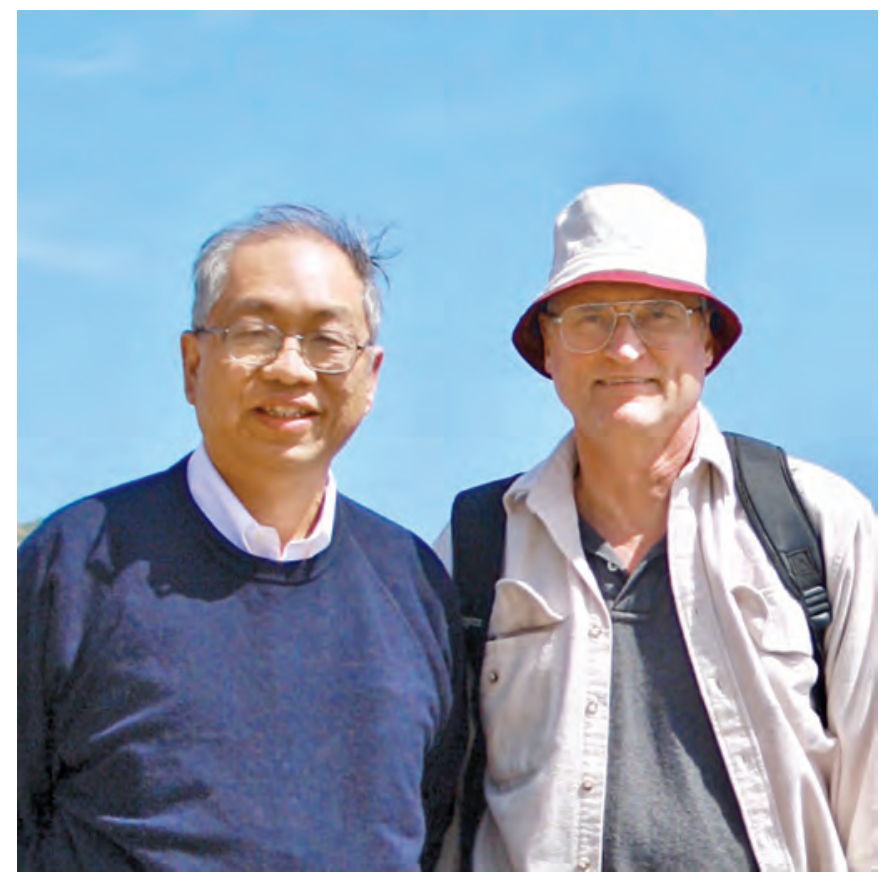

Figure 13. The collaborators Yau and Schoen. Yau writes that "more than half of my important papers were either written jointly with Rick or influenced by him."

In the following I shall share some reminiscences involving Rick that date back to the 1970s.

Both Simon and I arrived at Stanford in the fall of 1973; fortunately we had offices across the hallway

Shing-Tung Yau is William Caspar Graustein Professor of Mathematics at Harvard University. His email address is yau@math . harvard. edu. from each other. Rick Schoen was just starting out as a graduate student. A trenchant observation by Rick regarding the second variation formula for area functional for deformation of minimal hypersurfaces in Euclidean space led to our first collaboration, during which we managed to find a curvature estimate for stable minimal hypersurfaces up to dimension five. This result provided a direct proof of the famous Bernstein conjecture in these dimensions; it also turned out to be important for later work on the existence of mini-max hypersurfaces in a low-dimensional compact manifold.

This paper marked the beginning of our very successful collaboration over the last forty years. More than half of my important papers were either written jointly with Rick or influenced by him. We have a similar way of approaching problems in mathematics, and we are both excited by the power of nonlinear analysis in geometry, topology, and mathematical physics.

I was excited by the rigidity theorem of Mostow when I was a graduate student. I thought the theory of harmonic maps could offer a good approach for replacing the quasiconformal map argument of Mostow. (This was finally achieved in 1991 by Jost and me and also by Mok, Young, and Siu based on the similar strategy of Calabi.) I suggested to Rick that we explore the theory of harmonic maps as a complement to the theory of minimal surfaces. In the period of 1974 to 1975 , we studied the existence of harmonic maps and their geometric applications.

We exploited the uniqueness theory of harmonic maps when the image manifolds have nonpositive curvature in order to prove the rigidity of group actions on manifolds, reproving and generalizing some of the previous theorems in my thesis, as well as theorems pertaining to finite group actions on general manifolds. One extremely important development concerned the proof of the existence of harmonic maps from a Riemann surface into any manifold so long as there is a nontrivial map from the Riemann surface into the manifold inducing a nontrivial map on the fundamental group of the manifolds. This was remarkable because we were able to make sense of the action on the fundamental group for maps that are not necessarily continuous but have finite energy only.

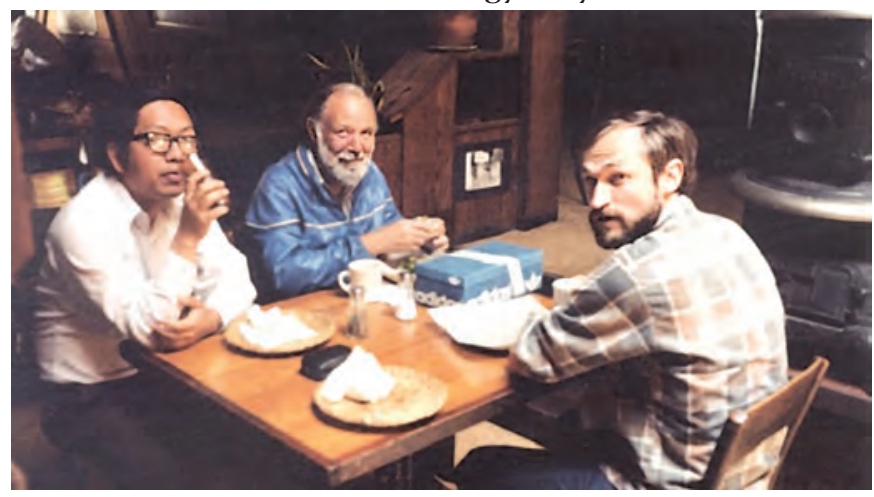

Figure 14. Yau and Schoen with Isadore Singer in San Diego, where they worked late into the night on their lecture series. 
We were led to believe that there should be some form of existence theorem for harmonic maps from the two-sphere. But before we started to work on that, we saw an announcement by Sacks and Uhlenbeck, who had proved such an existence theorem using ingenious ideas on treating the bubbling phenomena, and they can also treat the case with fundamental group. A couple of years later I, along with Siu, made use of the sphere theorem of Sacks-Uhlenbeck to prove the existence of rational curves in an algebraic manifold with positive bisectional curvature. The idea rests on a study of the second variational formula of minimal surfaces, similar to the case I studied with Rick, as mentioned above.

Rick and I also made use of these ideas to prove that the three-dimensional torus does not support a metric with positive scalar curvature. That had been a major open problem at the time. Our proof, in turn, led to Rick's and my subsequent proof of the positive mass conjecture in general relativity.

This theorem started the extensive applications of ideas from geometry and geometric analysis to general relativity. But the flow of ideas between geometers and general relativists can be very rewarding for all parties. I remembered that Hawking asked me to look into the four-dimensional analogue of the positive mass conjecture, which he and Gibbons called the positive action conjecture.

While pondering the problem posed by Hawking, Rick and I hit upon an amazing fact: the second variational formula of a minimal hypersurface can give rise to a conformal deformation of the minimal hypersurface, thus providing a way to achieve metrics of positive scalar curvature on the hypersurfaces. This important observation allowed us to do dimensional reduction as a way to understand higher-dimensional manifolds with positive scalar curvature. We were able to utilize such ideas to solve certain nonlinear equations, which are called the Jang equations in general relativity. These equations allow us, for example, to link the concept of black hole to mass in general spacetime. Rick and I were able to demonstrate rigorously that if the matter density is large in some fixed region, a black hole will form.

Since there is a regularity problem for minimal hypersurfaces for dimensions greater than seven, we have to restrict our theory to lower dimensions. Fortunately, about ten years ago we were able to remove the dimension restriction for the positive mass conjecture and also as pertains to the structure of manifolds with positive scalar curvature.

During our attempt to solve the positive mass conjecture, Rick and I naturally became interested in understanding the structure of manifolds with positive scalar curvature, which can, in turn, help us describe the structure of the topology of our universe. The first thing that Rick and I did was to determine what class of topology in a three-dimensional manifold can support metrics with positive scalar curvature. We found in early 1978 that it is possible to preserve metrics with positive scalar curvature among such manifolds. And we thought that it should be

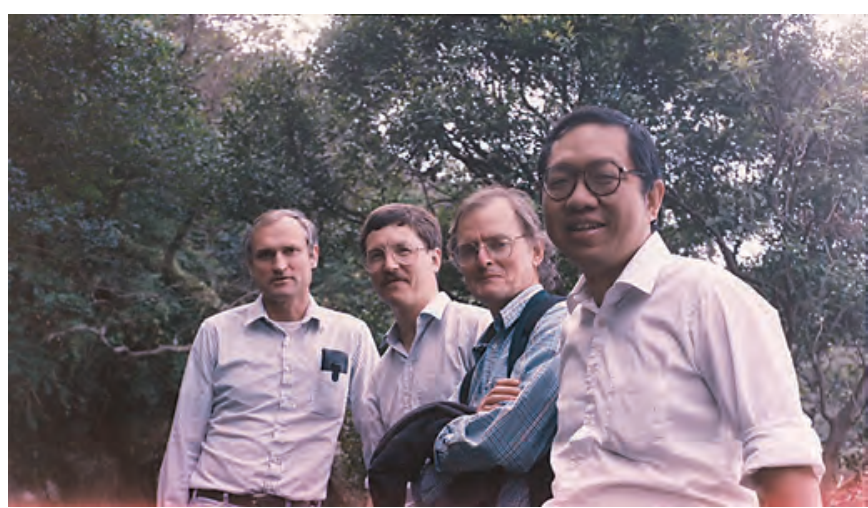

Figure 15. Richard Schoen, Robert Bartnik, Leon Simon, and Shing-Tung Yau.

possible to generalize the procedure to perform surgery on submanifolds of codimension 3 whose normal bundle is trivial.

I gave a plenary talk at the 1978 Helsinki Congress, where I discussed some of the results that Rick and I had obtained. On my way to Helsinki, I mentioned the surgery result to Blaine Lawson, who later worked with Gromov, using a more straightforward argument to reproduce a similar result. These results became the key to understanding the structure of manifolds with positive scalar curvature.

For me, working with Rick has been wonderful in so many ways. I remember that we spent the summer of 1979 together in Stanford while finishing the writing of our paper on the full positive mass conjecture. We worked during the day, often stopping to eat at a Chinese restaurant called Moon Palace. In the evening we stayed and swam in the house owned by a friend of Doris's, Rick's girlfriend and future wife. The weather was excellent, and we really had a good time.

Rick and I also gave a lecture series in Princeton, Berkeley, and San Diego, the contents of which were published in two books. We often went over the substance of these lectures a day before we delivered them. Some of the materials we presented were new, and sometimes our preparations lasted all the way to midnight and even beyond. Unfortunately not all of the materials were recorded or preserved. We can recall some of the statements that we could prove but not the full proof.

I've so far talked mainly about some of the work that Rick and I have done together. But he has, of course, made many spectacular achievements on his own, including the old conjecture of Yamabe, where Rick applied the positive mass conjecture in a brilliant manner. The subject of conformal deformation became an important area of research after this work by Rick. And he is clearly the leader of the whole field.

His proof with Brendle on the quarter-pinching of positively curved manifolds brought a dramatic resolution to a conjecture that had lasted for seventy years. All these accomplishments reveal the great insight and strength of Rick as a mathematician of tremendous originality. Rick 


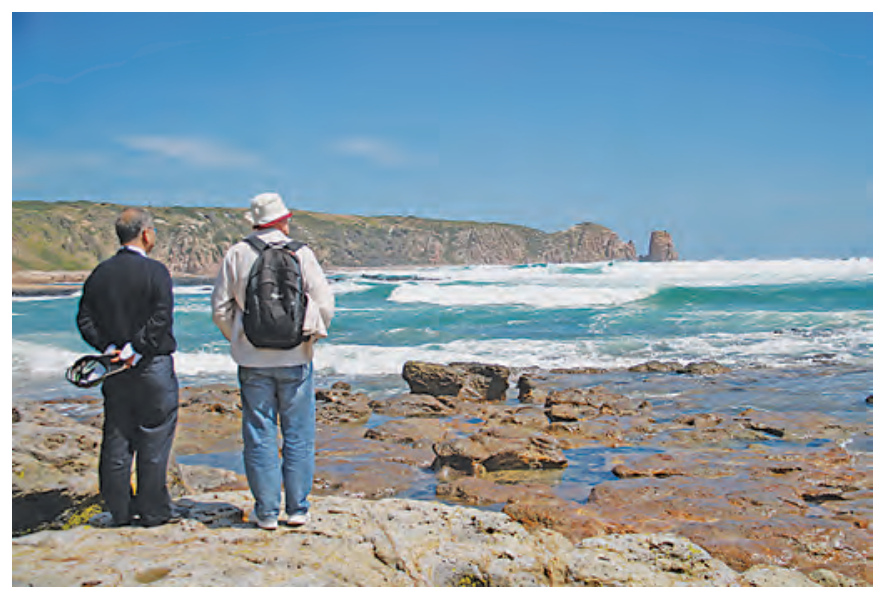

Figure 16.Yau and Schoen look out into the future.

has had many other important successes throughout his career that I could discuss here. But the work that I have discussed already demonstrates why Rick Schoen is a resoundingly worthy recipient of the Wolf Prize.

\section{William P. Minicozzi II}

\section{Schoen-Uhlenbeck Theory for Harmonic Maps}

In the early 1980s Schoen and Uhlenbeck proved a number of major results on harmonic maps in two papers that shaped the development of the field. The first paper [SU1] proved the crucial $\epsilon$-regularity, proved a sharp bound on the Hausdorff dimension of the singular set, and gave new insight into when singularities can occur. The second paper [SU2] proved complete boundary regularity and solved the Dirichlet problem for harmonic maps. These papers are extraordinarily clear and beautifully written, and the techniques remain important today.

A map $u: M^{m} \rightarrow N^{n}$ between Riemannian manifolds is harmonic if it is a critical point for the energy functional:

$$
E(u)=\int_{M}|\nabla u|^{2} .
$$

This is one of the most natural geometric variational problems. Taking $N^{n}=\mathbb{R}$ yields the special case of harmonic functions. Alternatively, taking $M^{m}=\mathbb{R}$ yields the special case of geodesics parametrized by a multiple of arclength. Taking $M^{m}=\mathbb{R}^{2}$ yields the special case of nice parametrizations of minimal surfaces. See Figure 17. Harmonic maps have played an important role in geometry, topology, and mathematical physics.

The energy $E(u)$ can be defined even when $u$ is not smooth; it suffices to have $|\nabla u|^{2}$ defined almost everywhere and integrable. Thus, harmonic maps can be defined weakly, and a central question becomes the regularity of a weak solution. A point $x$ is said to be regular if there is a neighborhood $B_{r}(x)$ where $u$ is smooth. The singular set $S$ is the complement of the regular set; it is automatically closed by definition. Both geodesics and harmonic functions are necessarily smooth, but general
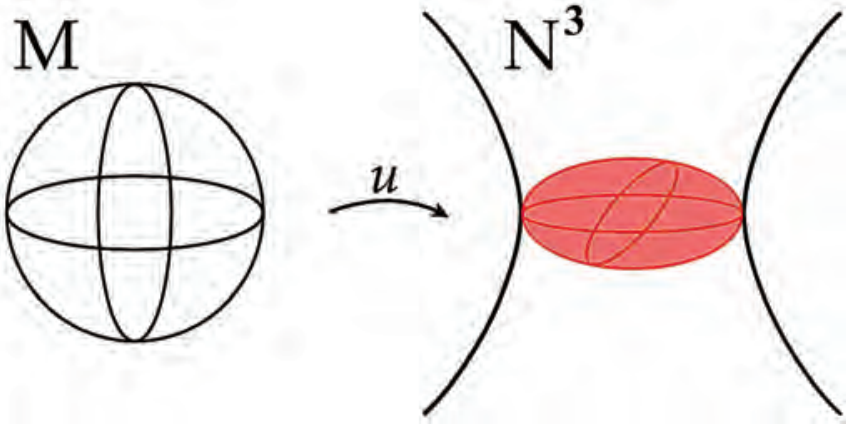

Figure 17. The standard map from $M=\mathbb{S}^{2}$ into the horizon at the neck of Schwarzschild space $N^{3}$ is a harmonic map as well as minimal.

harmonic maps-even ones that minimize energy-can have singularities.

In 1948 Morrey [M] showed that energy-minimizing harmonic maps from a surface $M$ must be Hölder continuous and therefore smooth as long as $M$ and $N$ are smooth. For the next thirty-five years, there were a number of results proving regularity for special classes of target manifolds $N$. For example, in the 1964 paper [ES, Eells-Sampson] proved the existence and regularity (smoothness) of harmonic maps when the target $N$ has nonpositive curvature. Hamilton solved the corresponding Dirichlet problem for manifolds with boundary.

There have been a number of great subsequent results on harmonic maps. Hardt $[\mathrm{H}]$ provides a nice survey.

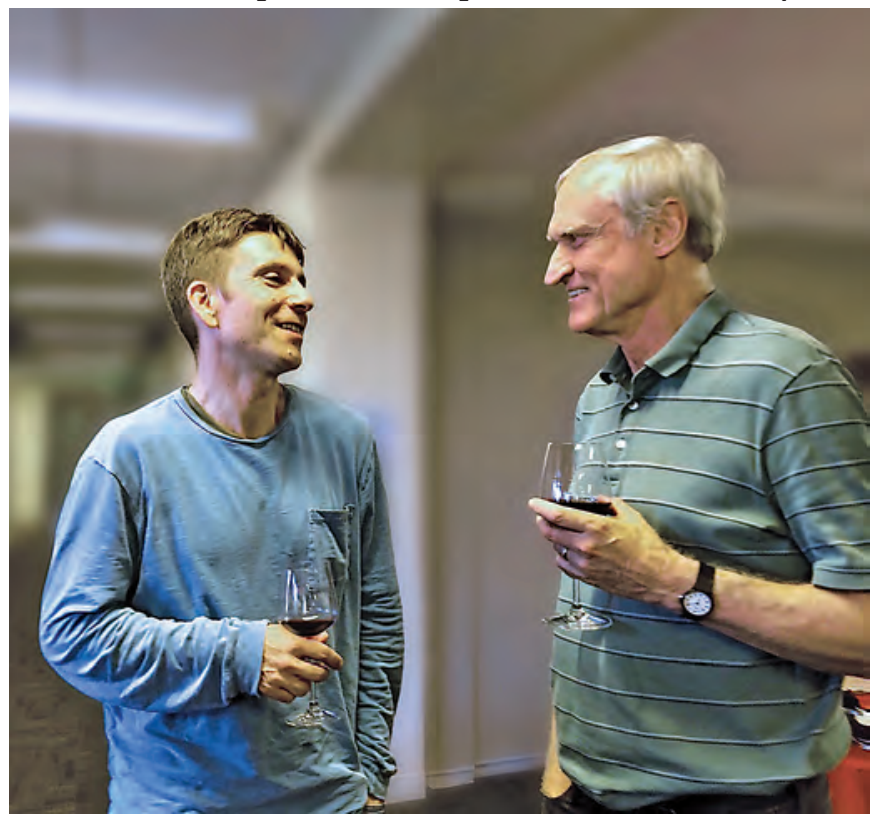

Figure 18. Minicozzi and Schoen in 2018.

The Size of the Singular Set

The simplest example of a singular harmonic map is the map

$$
\text { u : } M^{3}=B(0,1) \subset \mathbb{R}^{3} \rightarrow \mathbb{S}^{2}
$$


defined by

$$
u(x)=\frac{x}{|x|} .
$$

This map minimizes energy for its boundary values (e.g., among all maps that are the identity map when restricted to $\partial M^{3}=\mathbb{S}^{2}$ ). However it is clearly not continuous at the origin. Since it is smooth everywhere else, the singular set $S$ is the single point $\{0\}$. Notice that

$$
\operatorname{dim}(S)=0=3-3=\operatorname{dim}(M)-3,
$$

so we say $S$ has codimension 3 .

Schoen and Uhlenbeck showed that this is what happens in general:

Theorem 5 (Schoen-Uhlenbeck). If $u: M^{m} \rightarrow N^{n}$ is energy minimizing and $E(u)$ is finite and if $u(M)$ is contained in a compact subset of $N$, then

$$
\operatorname{dim}(S) \leq m-3 .
$$

If $m=3$, then $S$ is discrete.

An immediate corollary of Theorem 5 is that $u$ is entirely smooth when $M$ is a 2-dimensional surface. This special case was proven in 1948 by Morrey. Around the same time as Schoen and Uhlenbeck, Giaquinta and Giusti proved the special case of Theorem 5 when the image of $u$ is contained within a coordinate chart.

One of the central tools developed by Schoen and Uhlenbeck, and an absolutely fundamental result on its own, was the following $\epsilon$-regularity theorem:

Theorem 6. There exists $\epsilon>0$ so that if $u: M^{m} \rightarrow N^{n}$ is energy minimizing on the ball $B(p, r) \subset M$ for some $r \leq 1$ and

$$
r^{2-m} \int_{B(p, r)}|\nabla u|^{2} \leq \epsilon,
$$

then $u$ is Hölder continuous on $B(p, r / 2)$.

Schoen and Uhlenbeck actually proved something stronger than Theorem 6: there is a uniform Hölder bound that depends on $M, N, r$, and $\epsilon$ (and, in particular, not on $u$ ). Once the map $u$ is continuous then higher regularity theory shows that $u$ is smooth $[\mathrm{S}]$.

The quantity they estimate in Theorem 6 is the scaleinvariant energy:

$$
r^{2-m} \int_{B(p, r)}|\nabla u|^{2}=f_{B(p, r)}|\nabla u|^{2} / r^{2} .
$$

It is scale invariant in the sense that it does not change if we change $u$ by dilating the domain.

It follows immediately from the $\epsilon$-regularity theorem that each singular point $p$ has the property that

$$
r^{2-m} \int_{B(p, r)}|\nabla u|^{2}>\epsilon>0
$$

for $r$ arbitrarily small. When $m=2$, it follows immediately that there are only finitely many singular points. Namely, any ball, no matter how small, about a singular point contains energy at least $\epsilon>0$, so the number of singular points is at most the energy of $u$ divided by $\epsilon$.

In higher dimensions, Schoen and Uhlenback apply a covering argument to prove that

$$
\operatorname{dim}(S) \leq m-2 .
$$

Improving the dimension bound for $S$ to $m-3$ requires a more detailed blowup analysis, using monotonicity and scaling and a version of the Almgren-Federer dimension reduction argument of geometric measure theory. Energyminimizing maps have the following crucial monotonicity property: the scale-invariant energy is nondecreasing as a function of $r$ :

$$
r_{1}^{2-m} \int_{B\left(p, r_{1}\right)}|\nabla u|^{2} \leq r_{2}^{2-m} \int_{B\left(p, r_{2}\right)}|\nabla u|^{2} \text { for } r_{1}<r_{2} .
$$

This monotonicity fails for a general weakly harmonic map, though it does hold for an important class of harmonic maps called stationary harmonic maps.

Using the monotonicity, Schoen and Uhlenbeck show that at each $p$ there exists a tangent map,

$$
u_{p}: B(0,1) \subset T M_{P}=\mathbb{R}^{m} \rightarrow N,
$$

defined by blowing up about $p$ :

$$
u_{p}(x)=\lim _{\sigma_{i} \rightarrow 0} u\left(\exp _{p}\left(\sigma_{i} x\right)\right) .
$$

At a regular point $p$, the tangent map $u_{p}$ is trivial:

$$
u_{p}(x)=\lim _{\sigma_{i} \rightarrow 0} u(p)+g\left(\nabla u, \sigma_{i} x\right)=u(p) .
$$

In the simplest example where $u(x)=x /|x|$, we see that at $p=0$ the tangent map is

$$
u_{p}(x)=\lim _{\sigma_{i} \rightarrow 0} u\left(\sigma_{i} x\right)=\lim _{\sigma_{i} \rightarrow 0} \frac{\sigma_{i} x}{\left|\sigma_{i} x\right|}=u(x) .
$$

In general the tangent map is also homogeneous,

$$
u_{p}(x)=w_{p}(x /|x|) \text {, }
$$

and $w_{p}: \mathbb{S}^{m-1} \rightarrow N^{n}$ is harmonic.

This gives a criterion for regularity:

If there are no nontrivial harmonic maps from $\mathbb{S}^{k}$ to $N$ for every $k \leq m-1$, then every energyminimizing map from $B(0,1) \subset \mathbb{R}^{m}$ to $N$ must be smooth.

As a consequence there is a large class of target manifolds $N$ where the harmonic maps are smooth, including, for instance, $N$ with sectional curvature $K \leq 0$, as proven in Eells-Sampson.

\section{Boundary Regularity and the Dirichlet Problem}

The Dirichlet problem is the search for a harmonic map $u: M \rightarrow N$ with prescribed values of $u: \partial M \rightarrow N$ on the boundary of $M$. In the setting where $M$ is an interval this is equivalent to the problem of finding a length-minimizing geodesic between two points. When $M$ is a surface and the energy is, thus, conformally invariant, the Dirichlet problem is closely related to the Plateau problem for minimal surfaces. See Figure 19.

The 1982 results of Schoen and Uhlenbeck [SU1] give interior regularity for the solution $u$ but say nothing about regularity along the boundary. In 1983 Schoen and Uhlenbeck [SU2] proved complete boundary regularity for energy-minimizing maps: 


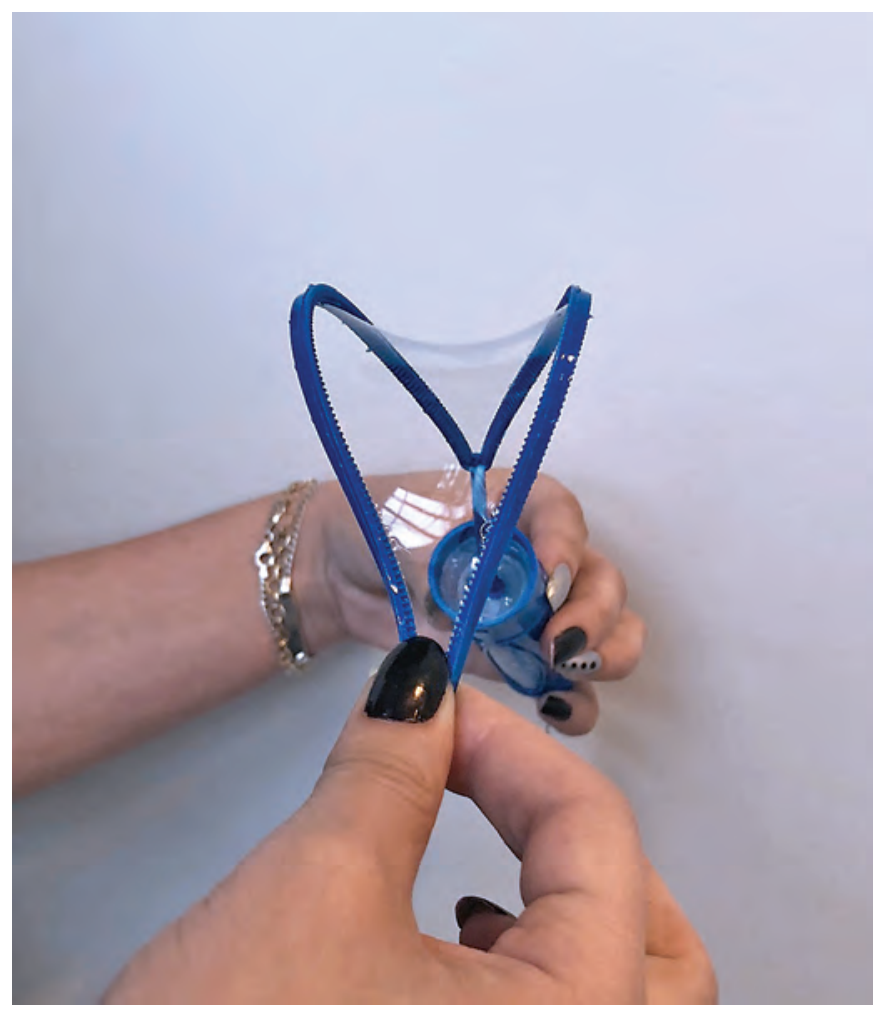

Figure 19. A soap film is a solution to the Dirichlet problem of finding a conformal harmonic map with prescribed boundary.

Theorem 7 (Schoen-Uhlenbeck). If $M$ is compact with $C^{2, \alpha}$ boundary, $u$ is energy minimizing (and $E(u)<\infty$ ), the image of $u$ is in a compact subset of $N$, and the restriction of $u$ to $\partial M$ is $C^{2, \alpha}$, then $u$ is $C^{2, \alpha}$ in a neighborhood of $\partial M$.

One consequence is that $S$ is a compact subset of the interior of $M$. The boundary regularity comes from the nonexistence of nontrivial smooth harmonic maps from the hemisphere which map the boundary to a point. Boundary regularity for surfaces was proven by Morrey in his work on the Plateau problem for minimal surfaces [M].

Using these results, Schoen and Uhlenbeck solved the Dirichlet problem.

Theorem 8 (Schoen-Uhlenbeck). Let $M$ be compact with $C^{2, \alpha}$ boundary as in Theorem 7 and suppose that $\partial N=\varnothing$. If $v: M \rightarrow N$ has

$$
\int|v|^{2}+|\nabla v|^{2}<\infty
$$

and the restriction of $v$ to $\partial M$ is $C^{2, \alpha}$, then there is an energy-minimizing harmonic map $u: M \rightarrow N$ that equals $v$ on $\partial M$. The singular set $S$ for $u$ is a compact subset of the interior of $M$ and

$$
\operatorname{dim}(S) \leq \operatorname{dim}(M)-3 .
$$

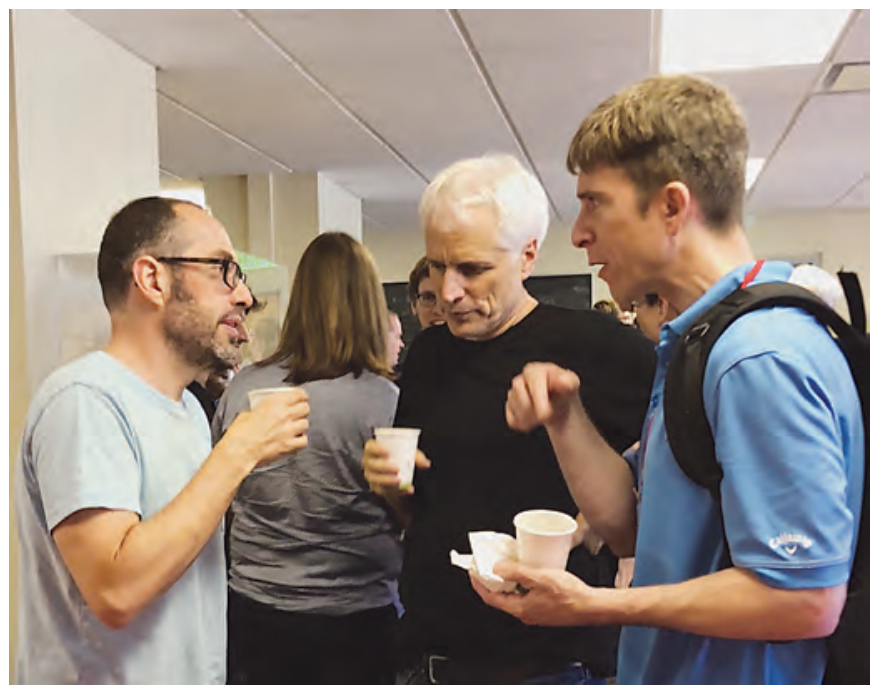

Figure 20. Schoen's students André Neves and Bill Minicozzi discussing mathematics as his long-time colleague, Brian White, listens at Stanford in 2018.

\section{References}

[ES] J. EELLS and J. SAMPSON, Harmonic mappings of Riemannian manifolds, Amer. J. Math. 86 (1964), 109-160 MR0164306.

[H] R. Hardt, Singularities of harmonic maps, Bull. Amer. Math. Soc. (N.S.) 34 (1997), no. 1, 15-34. MR1397098

[M] C. MORREY, The problem of Plateau on a Riemannian manifold, Ann. of Math. (2) 49 (1948), 807-851. MR0027137

[S] R. Schoen, Analytic aspects of the harmonic map problem. Seminar on nonlinear partial differential equations, MSRI Publ., 2, Springer, New York-Berlin, 1984, pp.321-358. MR0765241

[SU1] R. SCHOEN and K. UHLENBECK, A regularity theory for harmonic maps, J. Differential Geom. 17 (1982), no. 2, 307-335. MR0664498

[SU2] R. SCHOEN and K. UHLENBECK, Boundary regularity and the Dirichlet problem for harmonic maps. J. Differential Geom. 18 (1983), no. 2, 253-268. MR0710054

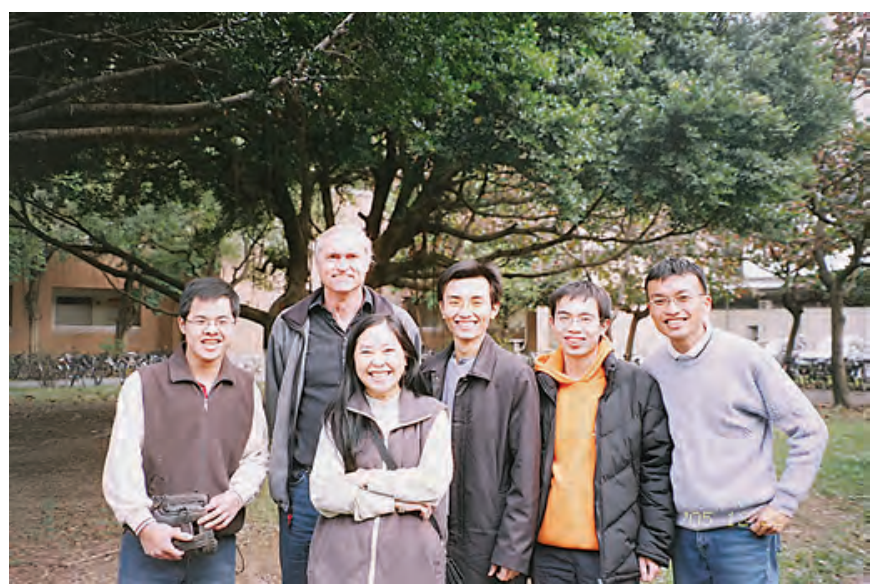

Figure 21. Rick Schoen with his doctoral student Yng-Ing Lee and her doctoral students in 2015. 


\section{Karen Uhlenbeck}

\section{Working with Rick Schoen}

The academic year 1979-80 at the Institute for Advanced Study in Princeton was a special year in differential geometry organized by S.-T. Yau. This year was indeed special for most of the participants, who met over a long period of time with their colleagues. They learned both classic and new open problems and a new set of techniques to apply to them. This period was a flowering of many methods of using nonlinear partial differential equations to approach problems in topology, differential geometry, and algebraic geometry. S.-T. Yau was a leader and driving force at this time. We all got to know each other, talk mathematics, and incidentally play volleyball and establish an eating co-op. Rick and I were both in residence for the year.

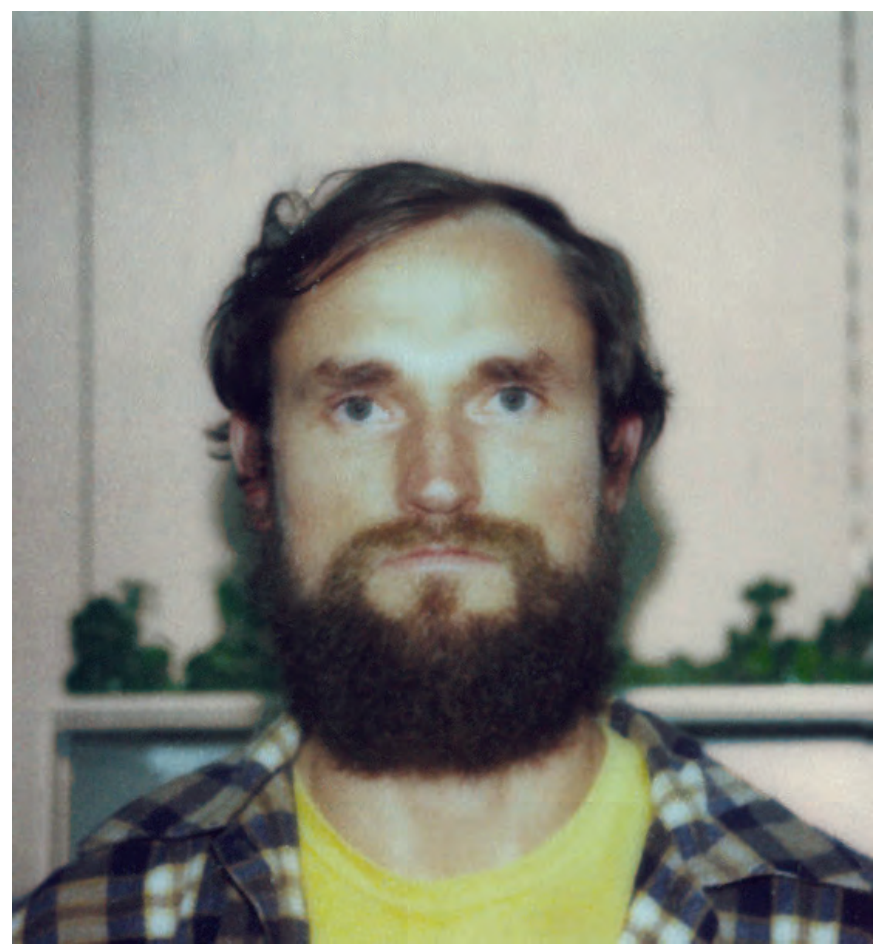

Figure 22. Schoen at IAS in 1979, where he first began working with Uhlenbeck on regularity using monotonicity.

I had since my student days been interested in minimization problems for maps between manifolds, and Rick brought to our collaboration a knowledge of minimal surface theory. Together we had a good working knowledge of nonlinear elliptic PDE. We did not start out with a deliberate goal of understanding harmonic maps, although we had seminars on them during the year. There were two background references we were both well aware

Karen Uhlenbeck is professor emerita at the University of Texas at Austin and visitor at the Institute for Advanced Study in Princeton. Her email address is uhlen@math. utexas. edu. of. A step in the construction of minimal surfaces in manifolds was the solution of the Dirichlet problem for harmonic maps of domains of the plane into arbitrary manifolds. Morrey's regularity theorem for minimizing the energy integral showed that the minimum of energy, which is by construction almost continuous, must lie in a Hölder space. We had both already used harmonic maps from surfaces to find minimal surfaces in manifolds. An already classic paper by Eells and Sampson from the 1960s discussed maps from an arbitrary dimensional space into manifolds of negative curvature, but their heat flow technique shed little light on harmonic maps into arbitrary manifolds from spaces of dimension greater than two. The standard regularity theorems only applied once one had a continuous solution, and direct minimization yields maps with only one derivative in $L^{2}$.

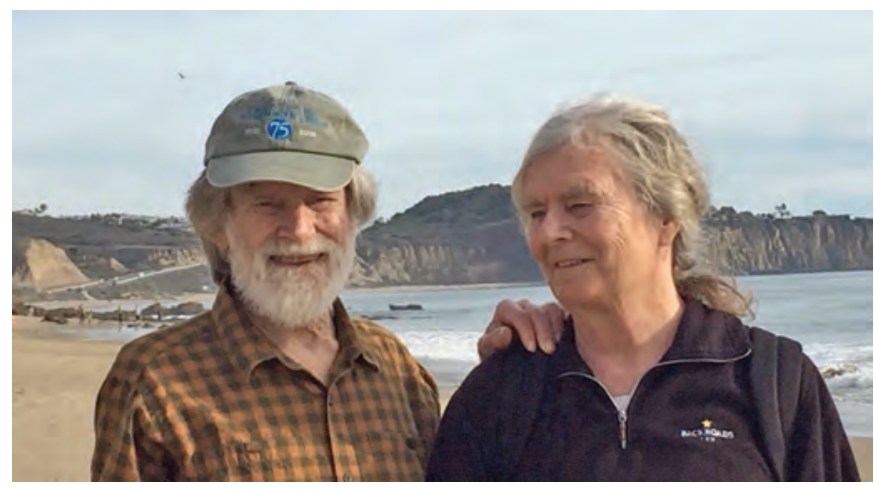

Figure 23. Uhlenbeck with her husband, the mathematician Bob Williams, in 2017.

At the very end of the year, Rick and I had an "aha moment" and our collaboration took off. We realized two things. First, the monotonicity formula for harmonic maps, which comes from variations by diffeomorphisms of the base manifold, showed that the minima were almost continuous. Minima lie in a Morrey or Besov space. Second, on balls of small energy, we could construct a comparison function to improve the estimate by smoothing out the boundary values a bit using mollifiers. So we got a modification of the classic theorem of Morrey to push us over into stronger estimates implying Hölder continuity. By covering and counting, we could show that the energy minimizer was smooth on the complement of a set of Hausdorff codimension 2.

In our papers, the monotonicity formula was the basis for all our arguments. We called it the scaling inequality, which is just as good a term, but not one that became accepted. I wonder today that neither Rick nor I nor the referee realized the accepted name for our basic estimate.

We also had a disagreement as to how to make the estimates. I wanted to construct a comparison minimizer, while Rick used the Euler-Lagrange equations and harmonic functions into the ambient manifold. He won, as in the later steps we did not assume minimization.

This was a great starting point. Later on, work of Tian et al. on Yang-Mills and work of Chang and Yang on 


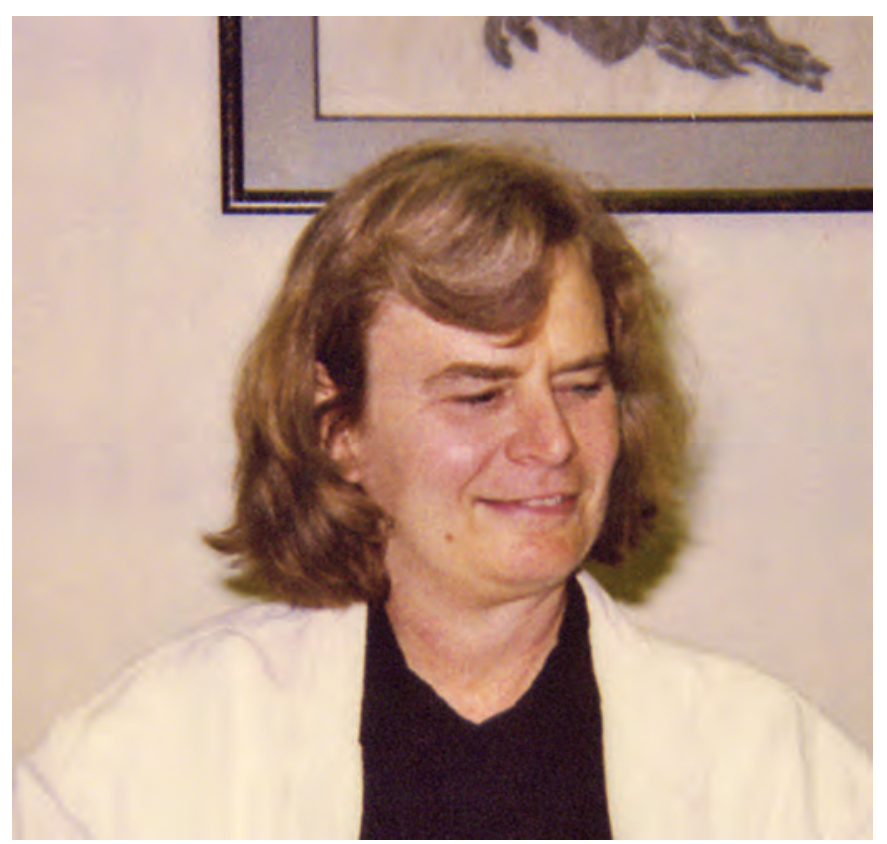

Figure 24. Uhlenbeck at the Institute for Advanced Study in 1995.

biharmonic maps between manifolds showed a similar result, but it is technically tricky to carry the result further in those cases.

During the next year, via long distance, we were able to refine this result. By letting the rescaling go to zero, one can easily construct a tangent map from $S^{n-1}$ to the image manifold, which has similar properties. In dimension 3, the tangent maps from $S^{2}$ to $N$ are smooth, and approximation of the map by a tangent map shows the singular set in $M$ consists of isolated points. Rick knew of a dimension-reducing argument of Federer in geometric measure theory. I was very anxious about this result but trusted Rick's confidence. The final result was that the singular set was of finite codimension-3 Hausdorff measure. We wrote two additional papers on Dirichlet boundary values and minimizing maps between spheres. I had become interested in the reduction of harmonic maps to ordinary differential equations using extra symmetry, and so I was pleased to be able to include these.

It was a pleasure to work with Rick. He brought valuable background information and consistent enthusiasm to our collaboration. The following image has stayed with me. Rick was until very recently a baseball player who played regularly. I have always thought that he brought his consistent practice of mathematics, insistence on carrying out the game to the end, and a real team spirit from baseball into mathematics.

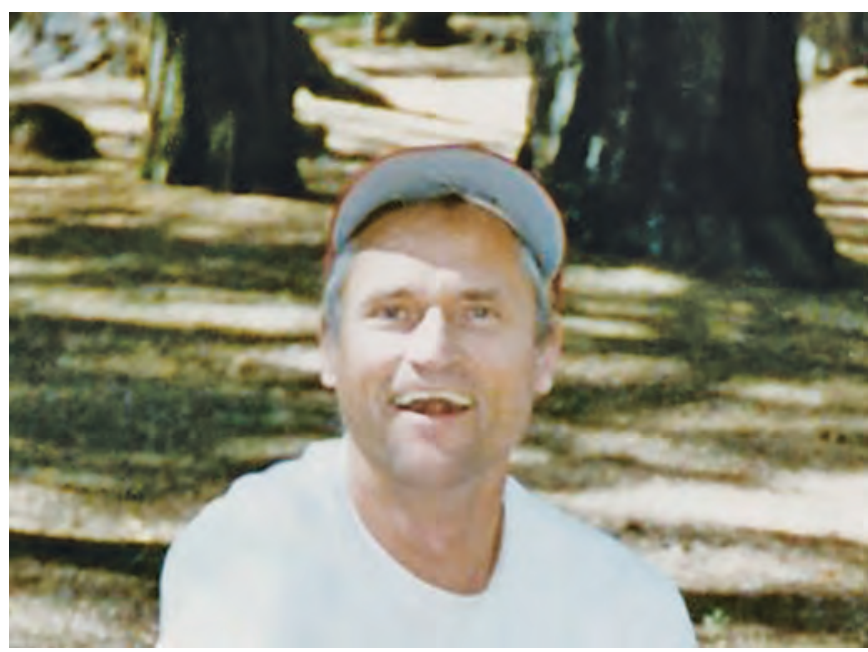

Figure 25. Uhlenbeck writes that Schoen brought "a real team spirit from baseball into mathematics" always "carrying out the game to the end."

\section{Rob Kusner}

\section{Classical Minimal Surfaces}

Following his work with Yau on the existence of incompressible minimal surfaces and the topology of 3-manifolds of positive scalar curvature and their breakthrough proof of the positive mass theorem in general relativity, Rick Schoen turned his attention to questions about classical minimal surfaces in $\mathbb{R}^{3}$.

The simplest complete minimal surface in $\mathbb{R}^{3}$ is the plane, and early in the twentieth century S. Bernstein characterized it as the only entire minimal graph. The generalization of this to higher dimensions became known as the Bernstein problem, which drove much work in the field, culminating in the 1968 Annals of Mathematics paper by Jim Simons and further explored in Rick's 1975 debut paper in Acta Mathematica with Leon Simon and Yau. Minimal graphs are stable-in fact, area-minimizinghypersurfaces. Both papers make use of the stability inequality from the second variation formula to bound the second fundamental form, leading to the solution of the Bernstein problem and to the regularity of such hypersurfaces (off a singular set of high codimension).

Even in $\mathbb{R}^{3}$ it's natural to ask whether the plane is the only complete stable surface. Schoen proved this with Doris Fischer-Colbrie in 1980 as a corollary to their results on complete stable surfaces in 3-manifolds of nonnegative scalar curvature [2]. A short proof of this generalized Bernstein theorem was also given by Manfredo do Carmo and C. K. Peng around the same time.

Another classical minimal surface problem concerns the uniqueness of the catenoid (see Figure 26). It can be motivated by a simple experiment. Imagine dipping a pair of rings into a soap solution. If the rings are

Rob Kusner is professor of mathematics at UMass Amherst. His email address is profkusner@gmai1.com. 


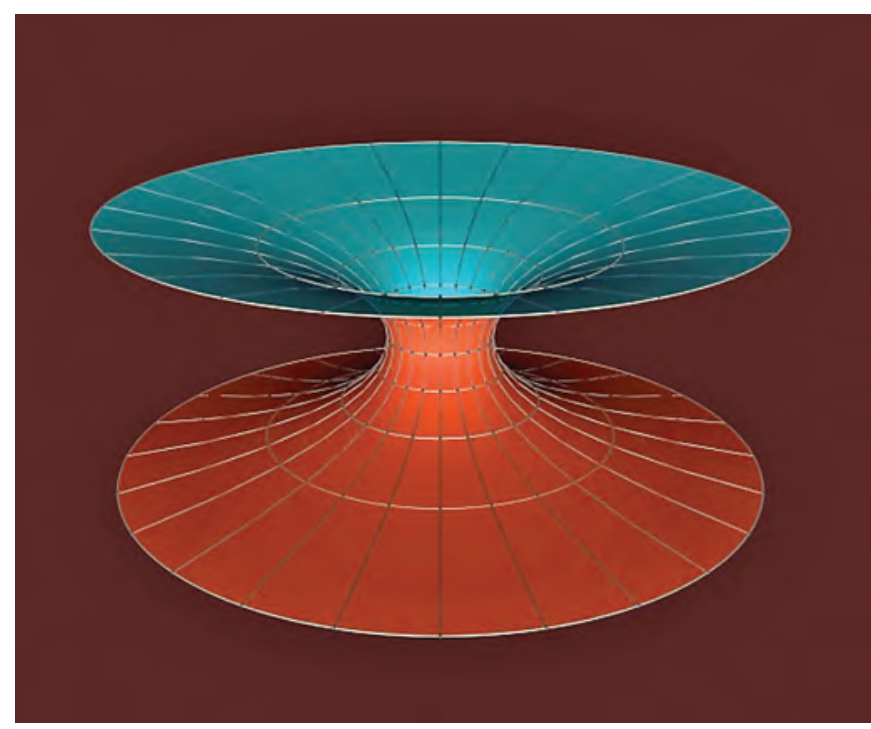

Figure 26. A catenoid is the only minimal surface of revolution (up to scaling and besides the plane).

close enough, they bound a connected least-area surface, a soap film, that is an embedded annulus (if the rings were linked, this annulus would be embedded with a full twist). In 1956 Max Shiffman proved a remarkable fact about this minimal annulus in case the boundary rings are perfectly round circles that lie in parallel planes: all the intermediate parallel planes must also meet the minimal annulus in round circles! This means the resulting minimal annulus must either be part of a catenoid (in the special case where the boundary circles are coaxial) or be part of a Riemann staircase (Figure 27).

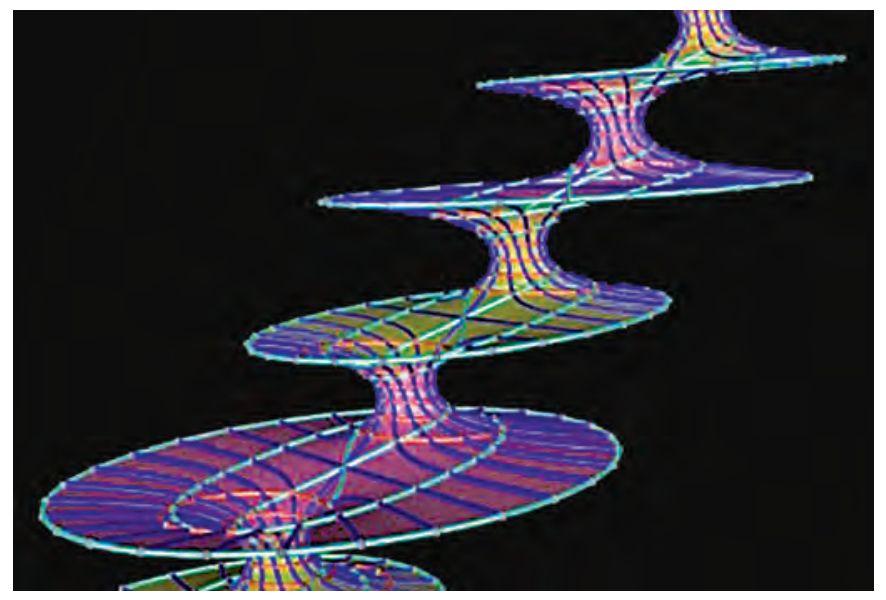

Figure 27. A Riemann staircase is a minimal surface foliated by round circles and straight lines (these come in a 1-parameter family, up to scaling).

More generally, if the boundary is a pair of convex curves in parallel planes, then Shiffman proved any minimal annulus bounded by them is still foliated by convex curves of intersection with the intermediate parallel planes.
Could there be another nonannular compact, connected minimal surface spanning a pair of convex curves in parallel planes?

Surprisingly, this remains an open problem, yet-in the spirit of the slogan "As all physicists know, and some mathematicians believe..."-the answer is conjectured (by Bill Meeks) to be "No."

The first answers in this direction were provided by Schoen in [4] in 1983. Applying the strong maximum principle of Eberhard Hopf and A. D. Alexandrov's "moving planes" reflection method, Schoen proved that any such minimal surface inherits the reflection symmetries of its boundary in the following sense: if the fundamental pieces of the boundary are "graphical" over the reflection planes, then so are the surface pieces. In particular, if there is a pair of orthogonal mirror planes carrying each such boundary curve into itself, then the minimal surface is necessarily annular (genus 0). See Figure 28.
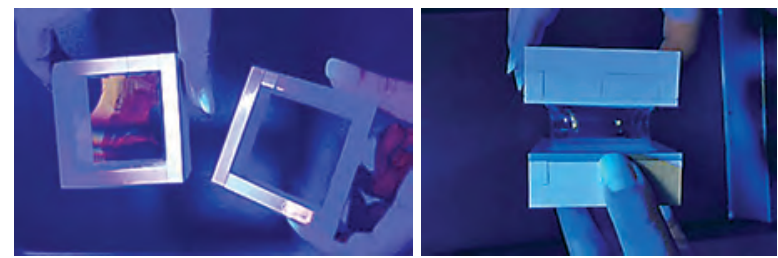

Figure 28. The connected minimal surface whose boundary is a pair of squares lying in parallel planes must be an annulus (genus 0 ).

Even for nonconvex boundary curves like the one in Figure 29 one can still get this graphical property for the mirror symmetric pieces of the surface as long as the boundary itself is composed of graphical pieces over the corresponding reflection plane, as in Figures 30-31, but in that case the surface could indeed have higher genus.

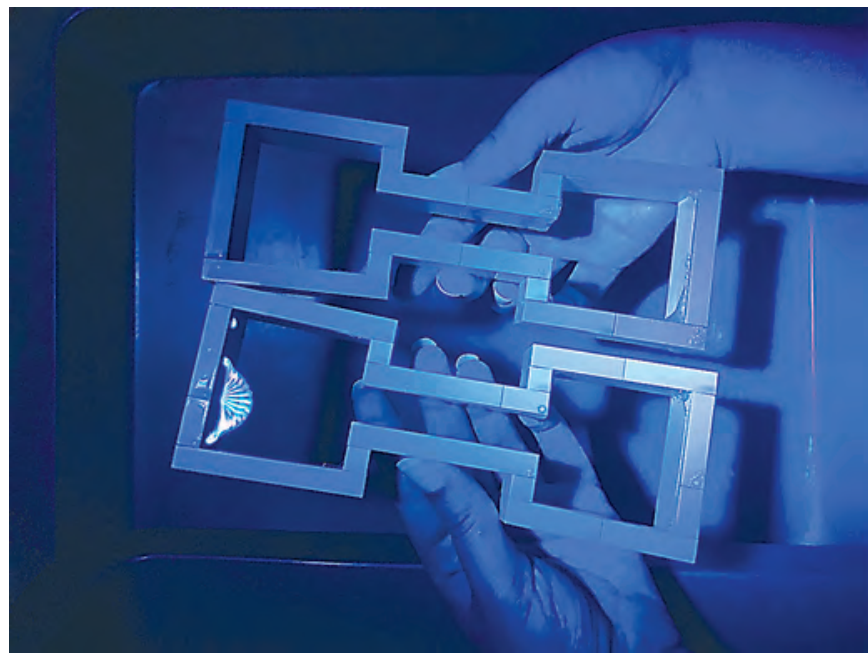

Figure 29. Connected minimal surfaces with these nonconvex boundaries are depicted in Figures 30-31. 


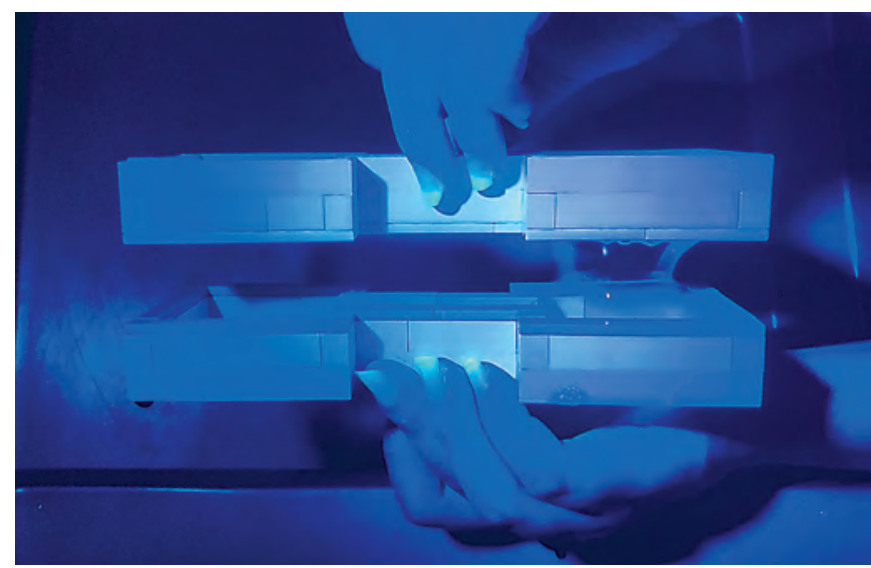

Figure 30. This connected minimal surface is an annulus (genus 0 ), which is a twice-punctured sphere.

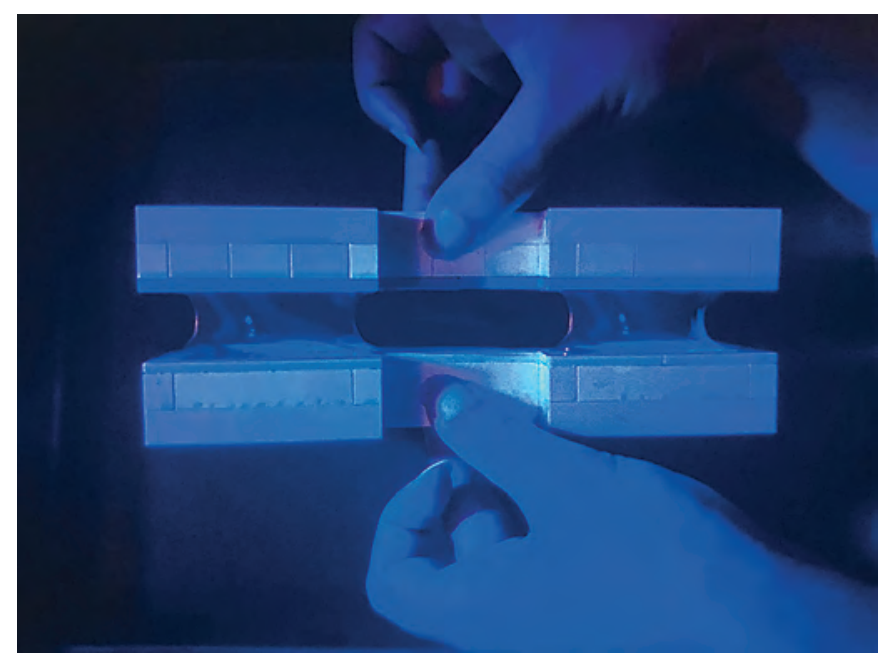

Figure 31 . This connected minimal surface is a twice-punctured torus (genus 1 ).

Schoen then considered the case of complete minimal surfaces without boundary; topological and geometric conditions on ends of the surface stand in for the boundary conditions. He introduced the notion of regular at infinity, meaning that each end of the minimal surface can be expressed as a graph having the asymptotic expansion (with respect to suitable coordinates $x, y, z$ for $\mathbb{R}^{3}$, with $x^{2}+y^{2}=r^{2}$ )

$$
z(x, y)=a \log r+\frac{b x+c y}{r^{2}}+O\left(\frac{1}{r^{2}}\right) .
$$

Physically, the logarithmic growth $a$ corresponds to the strength of the overall soap film surface-tension force pulling at that end; if it is nonzero, that end is asymptotic to a catenoid scaled proportionally to $a$ and is called a catenoidal end.

Using force balancing to align the ends and applying the Alexandrov reflection method, Schoen proved the following uniqueness theorem:
Theorem 9 (Schoen's Uniqueness Theorem [4]). The only complete, connected minimal surface in $\mathbb{R}^{3}$ with 2 ends, each regular at infinity, is the catenoid.

A decade later, Pascal Collin removed Schoen's "regularity at infinity" hypothesis. Meanwhile Korevaar, Solomon, and the author [3] proved the soap bubble analogue of this theorem: a complete, finite topology surface with 2 ends, properly embedded in $\mathbb{R}^{3}$ with constant mean curvature, must be a Delaunay unduloid (as in Figure 32).

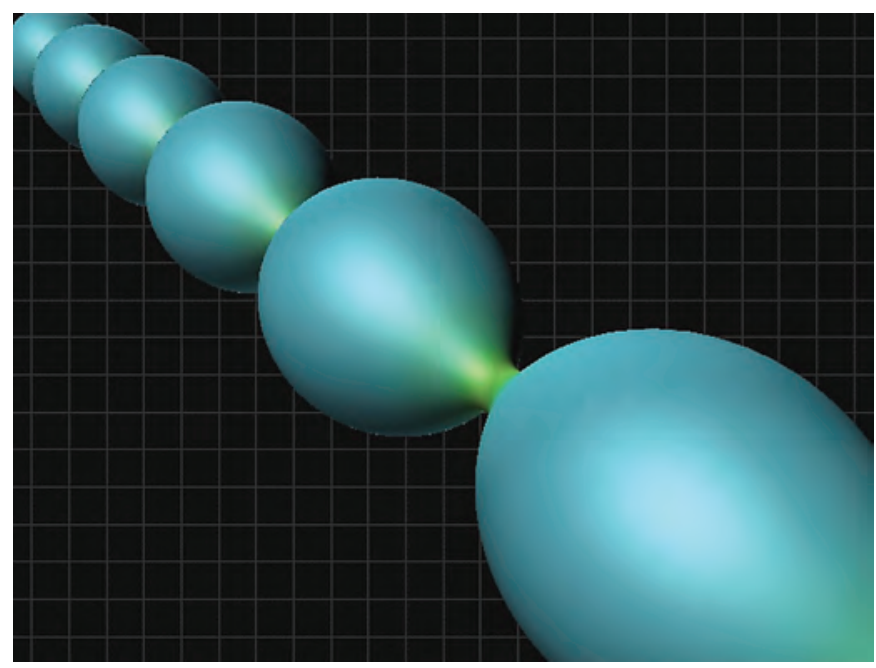

Figure 32. Korevaar, Kusner, and Solomon proved the soap bubble analogue of Schoen's soap film uniqueness theorem: a complete, finite topology surface with 2 ends, properly embedded in $\mathbb{R}^{3}$ with constant mean curvature, must be a Delaunay unduloid [3].

To get a sense of the amazing progress-the discovery of new examples and development of new methodssince 1983, note that Rick [4] remarked, "While there are a number of examples of finite total curvature surfaces known, some being regular at infinity, it is not known whether an embedded example exists besides the catenoid and the plane." Within a year, Celso Costa had mooted this, constructing the first example of such a minimal surface in more than two centuries!

The situation for infinite topology minimal surfaces in $\mathbb{R}^{3}$ is much more complicated. There are 1-ended examples like the singly periodic Scherk minimal surfaces (Figure 33) and the triply periodic Schwarz minimal surfaces of infinite genus. The latter can be constructed by rotating the "rectangular catenoid" in Figure 28 by a half-turn about each boundary edge. Both these examples come in families: changing the angle between the "wings" of the Scherk surfaces gives a 1-parameter family, while changing the edge ratio and separation of the rectangles gives a 2-parameter family of Schwarz surfaces.

For more than two ends, there are subtle topological obstructions discovered by Collin, Meeks, Rosenberg, and the author [1]: a complete, properly embedded minimal surface in $\mathbb{R}^{3}$ can have only countably many ends (like the 


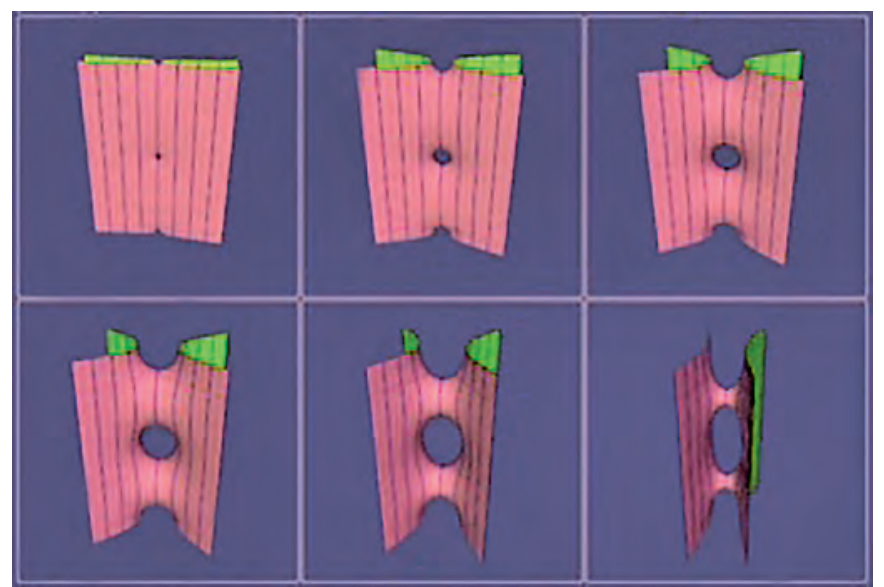

Figure 33. Infinite topology minimal surfaces include the singly periodic Scherk surfaces. These come in a 1-parameter family (up to scaling) determined by the angle between "wings" of the surface. We see a doubled plane or (suitably rescaled) catenoid in the limit as the angle tends to zero.

Riemann staircase in Figure 27, which has two limit ends and countably many middle ends).

Although Schoen's uniqueness theorem characterizes the catenoid topologically, it's also interesting to consider a coarser, measure-theoretic characterization, in terms of the density at infinity:

$$
\lim _{R \rightarrow \infty} \frac{\operatorname{Area}(\Sigma \cap B(0, R))}{\pi R^{2}},
$$

where $\Sigma$ is the minimal surface and $B(0, R)$ is a ball of radius $R$ in the ambient space.

Each end of a minimal surface that is regular at infinity has density 1 at infinity, and so the catenoid has density 2 at infinity. By monotonicity of area density, it can be seen that any minimal surface (besides a pair of planes) in $\mathbb{R}^{3}$ with density 2 at infinity must be embedded.

Are there any other minimal surfaces with density 2 at infinity besides the catenoid? There are! Indeed, the 1-parameter family of singly periodic Scherk surfaces (see Figure 33) also do, and the catenoid appears (upon suitable rescaling) in the limit as the "wing" angle tends to zero. It's conjectured that these are the only examples:

Conjecture 10. The catenoid and the family of singly periodic Scherk surfaces are the only complete, connected, embedded minimal surfaces in $\mathbb{R}^{3}$ with density 2 at infinity.

It's not clear who should be credited with this conjecture or even whether it is true. It first came up in a lunchtime conversation between Rick and me-the youngest of Rick's students from UC Berkeley-after we had all moved to UC San Diego for a few years in the mid-1980s. I recall Rick being characteristically agnostic but aware of the key issues, as if he'd thought about it for some time, and ready to share his insights with whomever he thought would have a good chance of solving the problem. Most of our interactions were like that, whether an

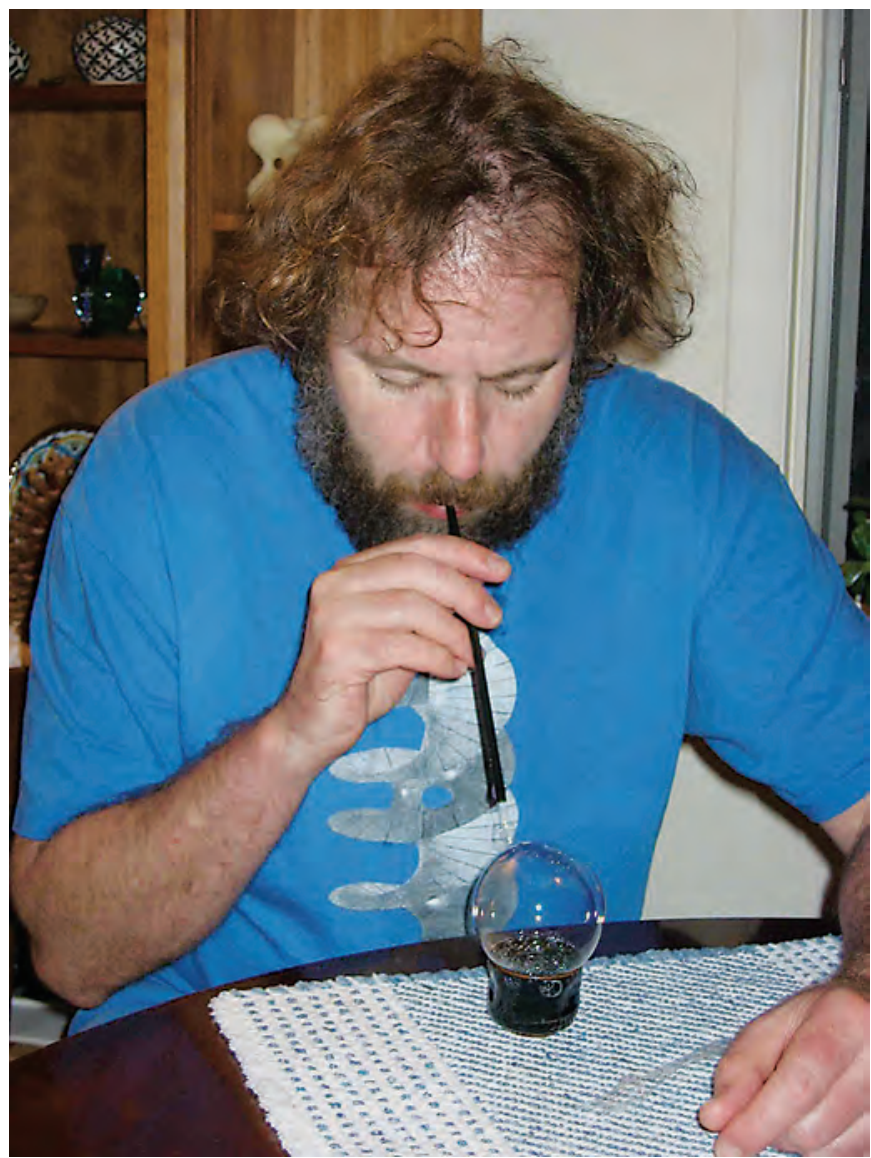

Figure 34. Kusner blowing an unduloidal soap bubble (at the end of the straw) in 2003.

hour-long phone call or a detailed handwritten letter (so 1980s, long before email), dropping by his office or out for a long lunch (preferably outdoors-so California, so not Massachusetts), on the volleyball or basketball court. Rick always treated me like a peer, letting me wander and find my own way, or occasionally like a teammate, providing gentle teasing which urges you to improve and simultaneously says he thinks you're tough enough to take it. No wonder Rick has had so many students. I'm grateful to be among them.

\section{Websites with Graphics:}

GANG: www.gang.umass.edu

Matthias Weber:www . indiana . edu/ minima1/archive/ GeometrieWerkstatt: service.ifam.uni-hannover.de / geometriewerkstatt/ga11ery/

\section{References}

[1] Pascal Collin, Robert Kusner, William H. MeeKs, and HAROLD ROSENBERG, The topology, geometry and conformal structure of properly embedded minimal surfaces, J. Differential Geom 67, (2004), 377-393. MR2153082 
[2] DORIS FISCHER-COLBRIE and RICHARD SCHOEN, The structure of complete stable minimal surfaces in 3-manifolds of nonnegative scalar curvature, Comm. Pure Appl. Math., 33 (1980), 199-211. MR0562550

[3] Nicholas J. KorevaAR, Rob KuSNER, and Bruce SOlOmon, The structure of complete embedded surfaces with constant mean curvature, J. Differential Geom. 30, (1989), 465-503. MR1010168

[4] Richard M. Schoen, Uniqueness, symmetry, and embeddedness of minimal surfaces. J. Differential Geom. 18 (1983), 791-809. MR0730928

\section{Fernando Codá Marques}

\section{The Yamabe Problem}

In 1984 Richard Schoen [3] solved the remaining cases of the famous Yamabe problem with the introduction of a remarkable idea. The Yamabe problem is the question of whether any given compact Riemannian manifold $\left(M^{n}, g\right)$ admits a conformal metric $\tilde{g}$ with constant scalar curvature. In two dimensions the answer is affirmative due to the uniformization theorem. In high dimensions the problem was proposed in 1960 by Yamabe [4], who mistakenly thought he had found a solution. Trudinger (1968) pointed out the error and fixed it when the scalar curvature is nonpositive. The case when $n \geq 6$ and the manifold is not locally conformally flat was proven in important work of Aubin (1976). Schoen finished the cases when $n=3,4$, or 5 or when $g$ is locally conformally flat in any dimension by discovering a surprising connection with the positive mass theorem of general relativity. We refer the reader to Lee and Parker [2] for a complete account of this history.

For $n \geq 3$, the Yamabe Problem is equivalent to finding a positive solution $u \in C^{\infty}(M)$ of the nonlinear elliptic partial differential equation

$$
\Delta_{g} u-c(n) R_{g} u+c(n) \lambda u^{\frac{n+2}{n-2}}=0,
$$

where $\Delta_{g}$ is the Laplace operator of $g, R_{g}$ is the scalar curvature of $g$,

$$
c(n)=\frac{(n-2)}{4(n-1)},
$$

and $\lambda \in \mathbb{R}$. Any positive solution $u$ gives rise to a conformal metric

$$
\tilde{g}=u^{\frac{4}{n-2}} g
$$

with constant scalar curvature $R_{\tilde{g}}=\lambda$.

This is in fact a variational problem. One can associate to every metric $\tilde{g}=u^{\frac{4}{n-2}} g$ in the conformal class $[g]$ of $g$ the Einstein-Hilbert action:

$$
\mathcal{R}(\tilde{g})=c(n) \frac{\int_{M} R_{\tilde{g}} d v_{\tilde{g}}}{\left(\int_{M} d v_{\tilde{g}}\right)^{\frac{n-2}{n}}} .
$$

Applying (1) and integrating by parts, we see that the Einstein-Hilbert action can also be written as

$$
\mathcal{R}(\tilde{g})=\frac{\int_{M}\left(\left|\nabla_{g} u\right|^{2}+\mathcal{c}(n) R_{g} u^{2}\right) d v_{g}}{\left(\int_{M} u^{\frac{2 n}{n-2}} d v_{g}\right)^{\frac{n-2}{n}}} .
$$

Fernando Codá Marques is professor of mathematics at Princeton University. His email address is coda@math. princeton . edu.

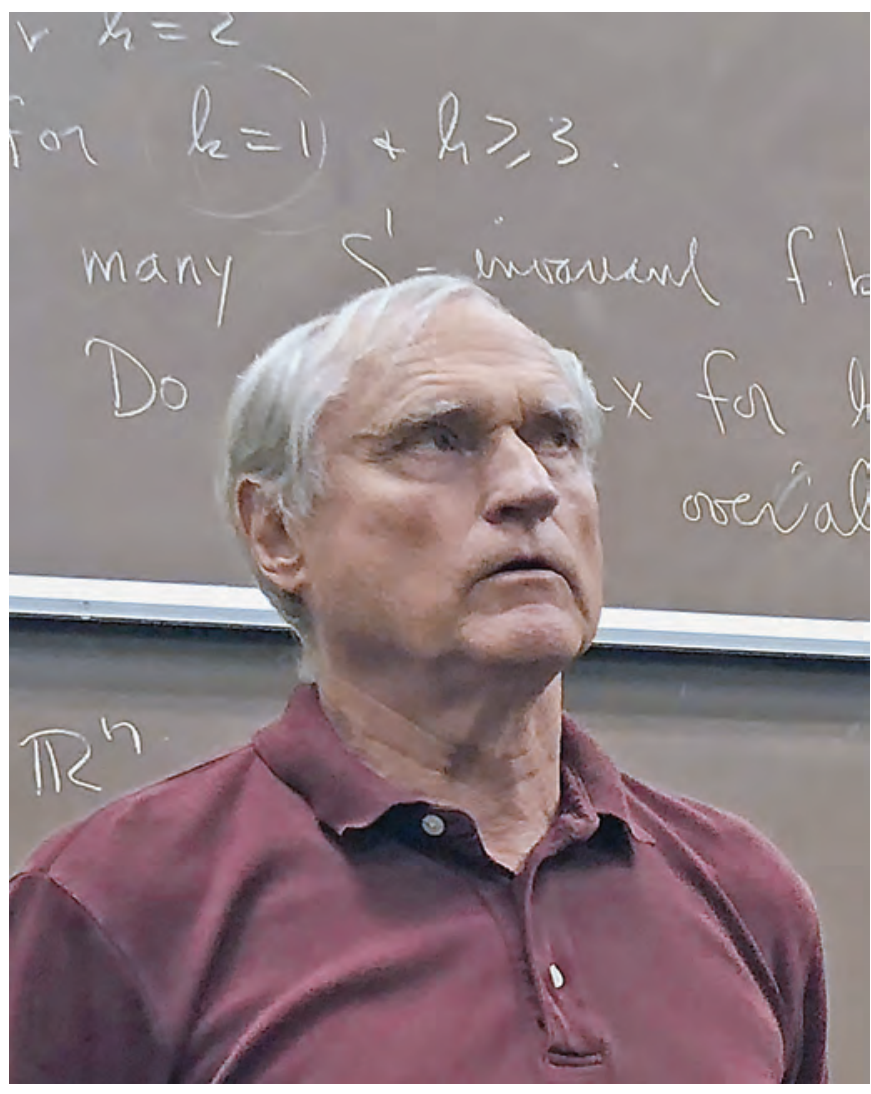

Figure 35. Schoen solved the Yamabe problem by discovering a surprising connection with the positive mass theorem.

This functional is clearly bounded from below. A metric $\tilde{g}$ is a critical point of $\mathcal{R}$ restricted to the conformal class $[g]$ if and only if $\tilde{g}$ has constant scalar curvature.

The Yamabe problem is hard because the equation (1) has a critical exponent for the Sobolev embeddings. The Sobolev space $W^{1,2}(M)$ is continuously embedded in $L^{\frac{2 n}{n-2}}(M)$ and is compactly embedded in $L^{q}(M)$ for every

$$
1 \leq q<\frac{2 n}{n-2} \quad \text { but not for } q=\frac{2 n}{n-2} .
$$

For this reason the direct method of the calculus of variations, which tries to extract a limit out of a minimizing sequence of the functional, is doomed to fail.

The difficulty can also be explained on the geometric side. If $\bar{g}$ denotes the round metric on $S^{n}$, then $\varphi^{*}(\bar{g})$ has constant scalar curvature equal to $n(n-1)$ for any diffeomorphism $\varphi$ of the sphere. If $\varphi$ is a conformal map, then

$$
\varphi^{*}(\bar{g})=u_{\varphi}^{\frac{4}{n-2}} \bar{g}
$$

for some positive solution $u_{\varphi}$ of the Yamabe equation (1) with $g=\bar{g}$ and $\lambda=n(n-1)$. It is also possible to prove that $u_{\varphi}$ is a minimizer of $\mathcal{R}$. The group

$$
\operatorname{Conf}\left(S^{n}, \bar{g}\right)
$$

of conformal diffeomorphisms of the round sphere is noncompact, and in fact for any given $p \in S^{n}$ one can 


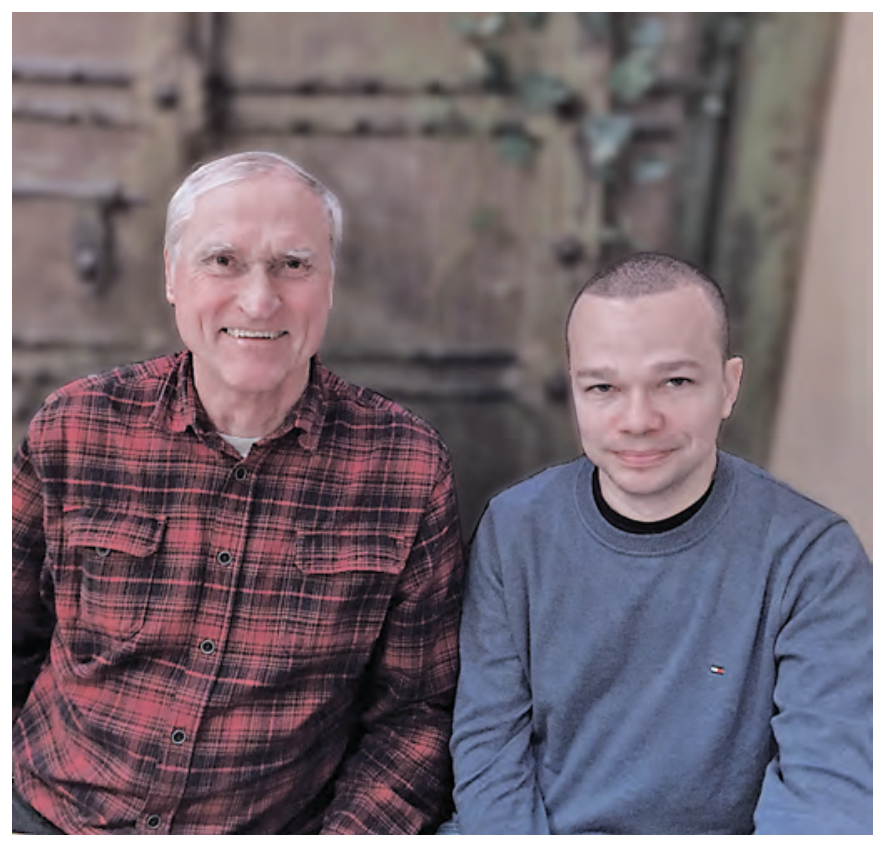

Figure 36. Schoen with his academic grandson, Fernando Codá Marques.

choose a sequence

$$
\varphi_{i} \in \operatorname{Conf}\left(S^{n}, \bar{g}\right)
$$

such that the corresponding functions

$$
u_{\varphi_{i}} \rightarrow 0
$$

in compact subsets of $S^{n} \backslash\{p\}$, while

$$
\sup _{B_{\varepsilon}(p)} u_{\varphi_{i}} \rightarrow \infty
$$

as $i \rightarrow \infty$ for any $\varepsilon>0$. When condition (2) holds for some sequence of solutions $u_{i}$ we say $u_{i}$ blows up at $p$.

The usual approach to the Yamabe problem consists of first finding a positive solution $u_{p}$ to the subcritical equation

with

$$
\Delta_{g} u-c(n) R_{g} u+c(n) \lambda u^{p}=0,
$$

$$
p<\frac{n+2}{n-2}
$$

which can be done by minimizing the corresponding functional and then studying the convergence of $u_{p}$ as

$$
p \rightarrow \frac{n+2}{n-2} \text {. }
$$

The Yamabe quotient of $(M, g)$, defined to be the conformal invariant

$$
Q(M, g)=\inf \{\mathcal{R}(\tilde{g}): \tilde{g} \in[g]\},
$$

plays a key role. Note that

$$
Q(M, g)=\inf _{u \in C^{\infty}(M)} \frac{\int_{M}\left(\left|\nabla_{g} u\right|^{2}+c(n) R_{g} u^{2}\right) d v_{g}}{\left(\int_{M} u^{\frac{2 n}{n-2}} d v_{g}\right)^{\frac{n-2}{n}}} .
$$

One always has

$$
Q(M, g) \leq Q\left(S^{n}, \bar{g}\right),
$$

as can be seen by choosing appropriate local test functions, and when the strict inequality

$$
Q(M, g)<Q\left(S^{n}, \bar{g}\right)
$$

holds, the subcritical solutions $u_{p}$ converge to a solution $u$ of (1). Hence in order to solve the Yamabe problem it suffices to prove that

$$
Q(M, g)<Q\left(S^{n}, \bar{g}\right)
$$

holds for any $(M, g)$ that is not conformally equivalent to the round sphere. If $n \geq 6$ and the manifold is not locally conformally flat, Aubin proved the strict inequality by constructing local test functions supported near a point where the Weyl tensor is nonzero.

The cases when $n=3,4,5$ or when $g$ is locally conformally flat in any dimension are more difficult because they require the construction of a global test function The key insight of Schoen was to realize that the test function would have to be Green's function $G_{p}$ of the conformal Laplacian

$$
L_{g}=\Delta_{g}-c(n) R_{g}
$$

smoothed out near its singularity $p$. One can suppose scalar curvature is positive, $R_{g}>0$, in which case Green's function is also positive. Since

$$
L_{g} G_{p}=0 \quad \text { outside } p,
$$

the conformal metric

$$
\hat{g}=G_{p}^{\frac{4}{n-2}} g
$$

has zero scalar curvature. The fact that, near $p$,

$$
G_{p}(x)=d(x, p)^{2-n}+\text { lower order terms }
$$

also implies that the metric $\hat{g}$ is asymptotically flat. See Figure 37.
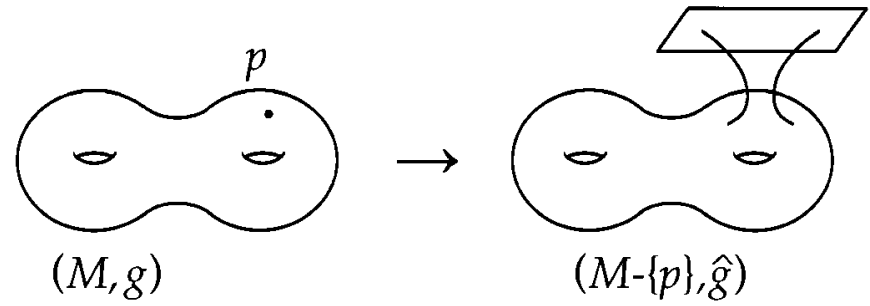

Figure 37. The main idea in Schoen's solution of the Yamabe problem is to invoke an asymptotically flat manifold obtained by blowing up the original manifold at a point. On the left is $(M, g)$ with the point $p$, and on the right is the blow-up, $(M-\{p\}, \hat{g})$, with an asymptotically flat end.

The mass $m$ of $\hat{g}$, as in the positive mass theorem, magically comes into play, and the strict inequality

$$
Q(M, g)<Q\left(S^{n}, \bar{g}\right)
$$

becomes a consequence of the positivity of $m$. The assertion that $m>0$ unless $\hat{g}$ is flat is the positive mass theorem.

In 1988, Schoen raised the question of whether the full set of solutions to equation (1) is compact in the $C^{k}$ topology (for any $k$ ) when the manifold is not conformally 


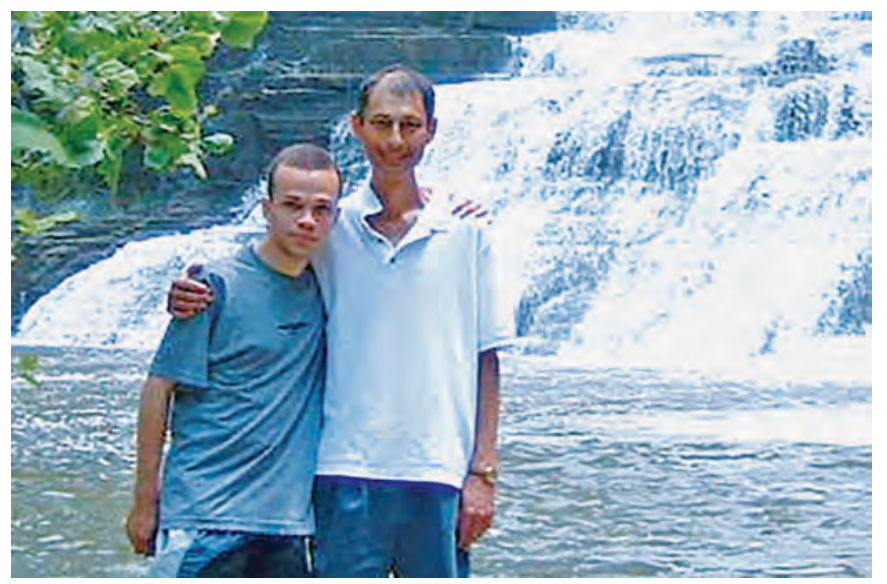

Figure 38. Fernando Codá Marques with his doctoral advisor, José Escobar, in 2003. Escobar completed his doctorate with Schoen in 1986 and is known in part for his work on the Yamabe problem with boundary.

equivalent to the standard sphere. This is basically a problem of establishing a priori estimates for the solutions. Schoen was motivated by a potential use of the Pohozaev identity and the positive mass theorem as obstructions to the blow-up phenomenon. The a priori estimates predicted that the Weyl tensor and its derivatives to order $[(n-6) / 2]$ should vanish at a blow-up point.

The a priori estimates were obtained much later for $n \leq 24$ in a paper [1] I wrote with Khuri and Schoen. They were known to hold for $n \leq 7$ (Druet, Y.Y Li, L. Zhang, Marques) and turned out to be false for $n \geq 25$ (Brendle, Brendle-Marques). In 2009, Khuri, Schoen, and I [1] discovered that the Pohozaev identity leads to a certain quadratic form (there is one for every dimension $n$ ) that is positive definite if $n \leq 24$ (it is not in higher dimensions), in which case we succeeded in proving the Weyl vanishing and the a priori estimates.

\section{On a Personal Note}

I had the privilege of learning directly from Rick during the academic year 2005-2006, when I visited Stanford University. It was a transformative experience in my career. I am indebted to him for having helped shape my vision of mathematics.

\section{References}

[1] M. A. Khuri, F. C. MArques, and R. M. Schoen, A compactness theorem for the Yamabe problem, J. Differential Geom. 81 (2009), no. 1, 143-196. MR2477893

[2] John M. LeE and Thomas H. PARKer, The Yamabe problem, Bull. Amer. Math. Soc. (N.S.) 17 (1987), no. 1, 37-91. MR0888880

[3] RICHARD SCHOEN, Conformal deformation of a Riemannian metric to constant scalar curvature, J. Differential Geom. 20 (1984), no. 2, 479-495. MR0788292

[4] Hidehiko Yamabe, On a deformation of Riemannian structures on compact manifolds, Osaka Math. J. 12 (1960), 21-37. MR0125546

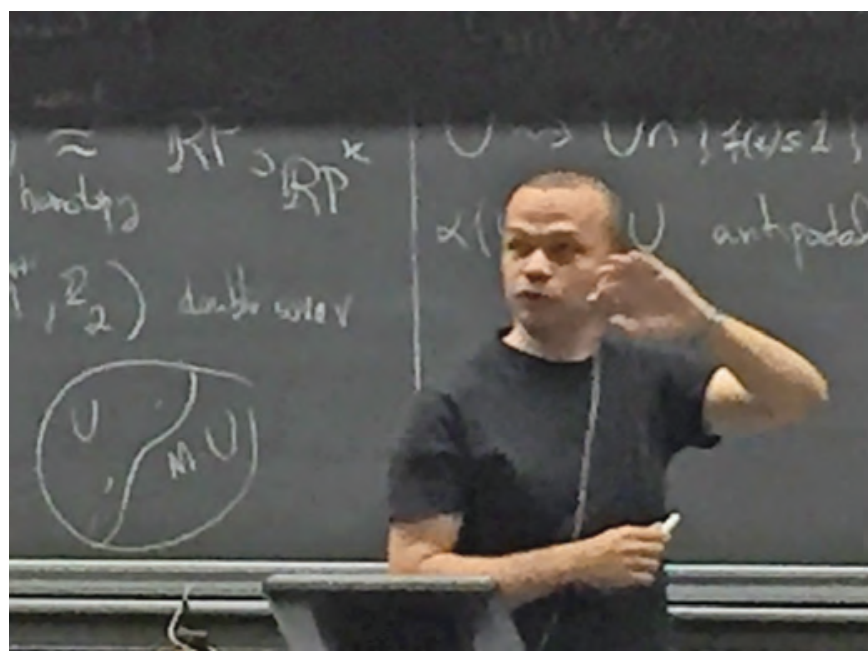

Figure 39. In addition to his important joint work with Schoen and Khuri on the Yamabe problem, Codá Marques is also famous for solving the Willmore conjecture with André Neves.

\section{Chikako Mese}

\section{Harmonic Maps in Singular Geometry and Rigidity}

Rick Schoen opened up a new frontier in geometric analysis by introducing harmonic maps in the singular setting through the series of papers [GS], [KS1], [KS2] written in collaboration with M. Gromov and N. Korevaar. More specifically, Rick initiated the study of harmonic maps into nonpositively curved metric spaces (NPC spaces) and introduced new techniques that were used to settle outstanding questions in rigidity problems. Here we will describe this work and further applications.

One of the main applications of harmonic maps is in geometric rigidity. Let $M$ be a compact or a finite volume Riemannian manifold with universal cover $\tilde{M}$, and let $\rho: \pi_{1}(M) \rightarrow \operatorname{Isom}(X)$ be a homomorphism from the fundamental group of $M$ into the group of isometries of a contractible metric space $X$. We say this setup is rigid if there exists a totally geodesic map $u: \tilde{M} \rightarrow X$ that is $\rho$-equivariant:

$$
u(\gamma(x))=\rho(\gamma)(u(x)), \quad \forall \gamma \in \Gamma, \forall x \in \tilde{M} .
$$

Some of the most influential rigidity results concern special cases of this setup. In the original Mostow rigidity theorem, $\tilde{M}$ and $X$ are both $n$-dimensional hyperbolic manifolds with $n \geq 3$, and $\rho$ is a discrete, faithful, and co-compact isometric action. Mostow also generalized this result by considering cases when $\tilde{M}=G / K$ and $X$ are other symmetric spaces. The celebrated Margulis superrigidity further extended Mostow's work by allowing

Chikako Mese is professor of mathematics at Johns Hopkins University. Her email address is cmese@math. jhu. edu. 
for a more general homomorphism $\rho$ and established the arithmeticity of lattices $\Gamma$ in groups $G$ when

$$
\operatorname{rank}(G / K) \geq 2 \text {. }
$$

The rank is the dimension of the maximal Euclidean space that can be isometrically embedded in $\tilde{M}=G / K$. Margulis also proved the higher rank non-Archimedean superrigidity by considering lattices in $p$-adic groups which, unlike their Archimedean counterpart, do not act on smooth Riemannian manifolds but instead act on Bruhat-Tits' Euclidean buildings. Euclidean buildings form an important class of NPC spaces, which we define in the next paragraph.

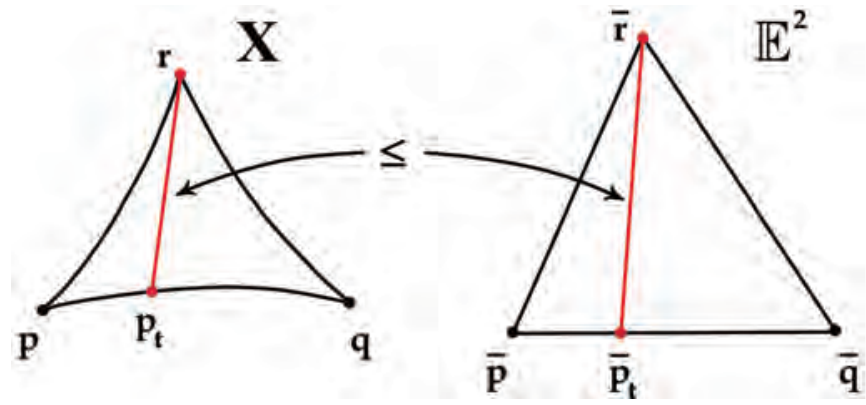

Figure 40. A geodesic space $X$ is an NPC space if every geodesic triangle in $X$ is thinner than a comparison geodesic triangle in the Euclidean plane $\mathbb{E}^{2}$.

A complete metric space $(X, d)$ is a geodesic space if every pair of points $p, q \in X$ is joined by a geodesic whose length is the distance between the points. Ageodesic space $(X, d)$ is an NPC space if it also has nonpositive curvature in the sense of triangle comparison: a geodesic triangle in $X$ with vertices $p, q, r$ is thinner than a comparison geodesic triangle in Euclidean 2-plane $\mathbb{E}^{2}$ (as in Figure 40). More precisely, if $\bar{p}, \bar{q}, \bar{r} \in \mathbb{E}^{2}$ are such that

$$
d(p, q)=|\bar{p}-\bar{q}|, d(q, r)=|\bar{q}-\bar{r}|, d(r, p)=|\bar{r}-\bar{p}|,
$$

then

$$
d\left(p_{t}, r\right) \leq|((1-t) \bar{p}+t \bar{q})-\bar{r}|,
$$

where $t \mapsto p_{t}$ for $t \in[0,1]$ is the constant speed parameterization of the geodesic from $p$ to $q$. The study of NPC spaces (more generally, spaces with curvature bounded from above by $\kappa$ known as CAT $(\kappa)$ spaces) was initiated by the foundational work of A. D. Alexandrov and further brought to prominence by Gromov.

Hadamard manifolds (complete and simply connected Riemannian manifolds with sectional curvatures bounded from above by 0 ) are examples of NPC spaces. A simple example of an NPC space that is not a manifold is a tripod $T$ that is the union of three copies of the interval $[0, \infty)$ identified at zero, as in Figure 41. The distance between two points $a$ and $b$ lying in different copies of $[0, \infty)$ is defined to be $a+b$. Note that any pair of points in $T$ is contained in a totally geodesic isometric embedding of $\mathbb{R}$ into $T$.

An $n$-dimensional Euclidean building $X$ is a more complicated example of an NPC space, but one can think of such buildings as higher-dimensional versions of the

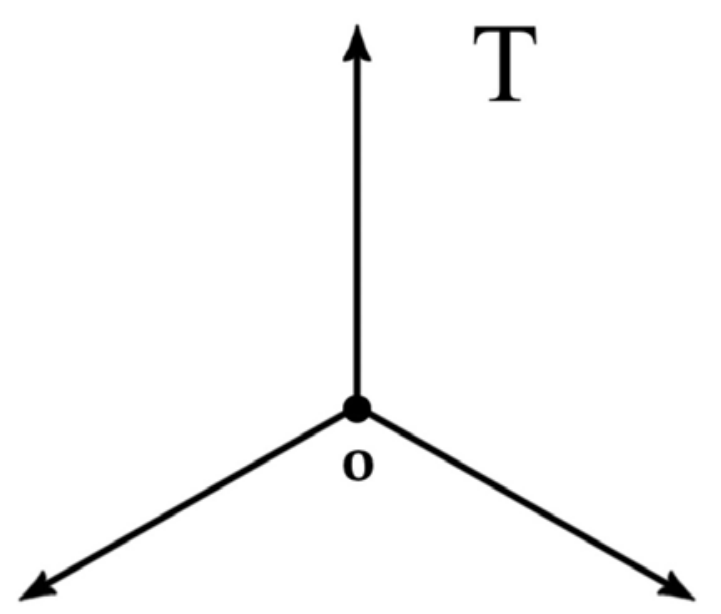

Figure 41. The tripod, $T$, is an example of an NPC space that is not a manifold.

tripod in the sense that these metric spaces have the property that any two points $p, q \in X$ are contained in an image of a totally geodesic and isometric embedding of $\mathbb{E}^{n}$ in $X$. However, not all NPC spaces have this property. Other important examples come from different areas of mathematics, such as geometric group theory and Teichmüller theory. For example, hyperbolic buildings arise in the study of hyperbolic groups of Gromov, and the Weil-Petersson completion of a Teichmüller space of a surface of genus $\geq 2$ is an NPC space.

Korevaar and Schoen [KS1], [KS2] consider maps $u$ : $M \rightarrow X$ from an $m$-dimensional Riemannian manifold, $m$, to an NPC space, $X$. For simplicity, we will let $M$ be a compact Lipschitz domain in Euclidean space. To define energy, consider the square of the difference quotient at a point $x \in M$ and integrate it over unit vectors $V$ to define an $\varepsilon$-approximate energy density function

$$
e_{\varepsilon}(x)=\int_{\mathbb{S}^{m-1}}\left(\frac{d(u(x), u(x+\varepsilon V))}{\varepsilon}\right)^{2} d \sigma(V) .
$$

When the measures $e_{\varepsilon}(x) d x$ have uniformly bounded total mass then they converge weakly as $\varepsilon \rightarrow 0$ to a measure of the form $e(x) d x$ where $|\nabla u|^{2}:=e(x)$ is an integrable function. We define the energy functional by setting

$$
E(u)=\int_{M}|\nabla u|^{2} d x .
$$

In this way, one can define the Sobolev space $W^{1,2}(M, X)$ (and in a similar way, $W^{1, p}(M, X)$ for $p>1$ and $B V(M, X)$ for $p=1$ ) and the notion of energy-minimizing maps.

Gromov-Schoen and Korevaar-Schoen proved the existence of energy-minimizing maps for the Dirichlet 
problem and certain equivariant problems. Of particular importance to rigidity is that energy-minimizing maps are essentially unique (e.g., a $\rho$-equivariant energyminimizing map is unique if $X$ is negatively curved but may only be unique up to parallel translation along a flat subspace generally).

The uniqueness follows from quadrilateral comparison inequality (a special case of Reshetnyak's theorem):

$$
\begin{aligned}
d^{2}\left(m_{p, s}, m_{q, r}\right) \leq & \frac{1}{2} d^{2}(p, q)+\frac{1}{2} d^{2}(r, s) \\
& -\frac{1}{4}(d(p, s)-d(q, r))^{2}
\end{aligned}
$$

for the ordered sequence $\{p, q, r, s\} \subset X$ where $m_{x, y}$ is the midpoint between $x$ and $y$. See Figure 42 .

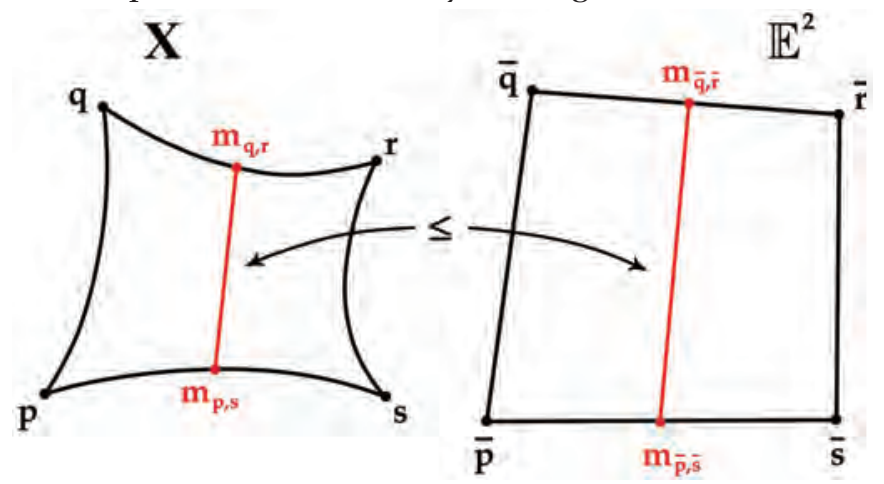

Figure 42. A quadrilateral comparison implies uniqueness for energy-minimizing maps.

This in turn implies the convexity of the energy functional:

$$
\begin{aligned}
\int\left|\nabla\left(\frac{u+v}{2}\right)\right|^{2} d x \leq & \frac{1}{2} \int|\nabla u|^{2} d x+\frac{1}{2} \int|\nabla v|^{2} d x \\
& -\frac{1}{4} \int|\nabla d(u, v)|^{2} d x
\end{aligned}
$$

where $u, v \in W^{1,2}(M, X)$ and

$$
\left(\frac{u+v}{2}\right)(x)=m_{u(x), v(y)} .
$$

Note that if $u$ and $v$ are energy minimizing, then the map $\frac{u+v}{2}$ is also energy minimizing and $d(u, v)$ is a constant.

The uniqueness of energy-minimizing maps suggests that if one wants to prove rigidity, then the first step should be to find a $\rho$-equivariant energy-minimizing map and then try to prove that it is totally geodesic. (This idea is mentioned in Yau's contribution to this article as the motivating source for his work with Schoen on harmonic maps.) Indeed, building upon the work of Y.-T. Siu and K. Corlette, Gromov and Schoen used harmonic maps in the singular setting to study the $p$-adic case of the superrigidity problem and established nonArchimedean superrigidity and consequent arithmeticity for lattices of certain rank-1 groups. A crucial issue in this application is to understand the regularity and singularity of harmonic maps. Natural questions that arise are: Under what conditions are harmonic maps regular? If they are not regular, what can we say about the structure of the singular set?

Korevaar and Schoen proved the following regularity theorem:
Theorem 11. An energy-minimizing map $u:(\Omega, g) \rightarrow$ $(X, d)$ from a Lipschitz Riemannian domain into an NPC space is locally Lipschitz continuous, where the Lipschitz constant of $u$ at $x \in \Omega$ is dependent on the geometry of $(\Omega, g)$, the distance of $x$ to $\partial \Omega$, and the total energy of $u$.

On the one hand, Lipschitz regularity is the optimal result when the target is assumed to be only an NPC space. On the other hand, the crucial idea in Gromov-Schoen [GS] is that energy-minimizing maps behave better than in Theorem 11 when the target has a certain manifold structure.

For a map $u: \Omega \rightarrow X$ into an NPC space, define the regular set $\mathcal{R}(u)$ of $u$ as the set of points in $\Omega$ that possess a neighborhood mapping into an image of a totally geodesic and isometric embedding of a smooth Riemannian manifold and define the singular set $S(u)$ as its complement. For example, the leaf space of the vertical foliation of the quadratic differential $z d z^{2}$ on $\mathbb{C}$ endowed with the distance function defined by the vertical measure is isometric to the tripod $T$ (see Figure 43). The natural projection map

$$
u: \mathbb{D} \rightarrow \mathrm{T}
$$

from the unit disk centered at 0 is an energy-minimizing map. It takes any neighborhood away from the origin into at most two copies of $[0, \infty)$. Thus, $u$ is locally a harmonic function away from the origin. The image of any neighborhood of the origin is not a manifold. Consequently, $\mathcal{R}(u)=\mathbb{D} \backslash\{0\}$ and $S(u)=\{0\}$.

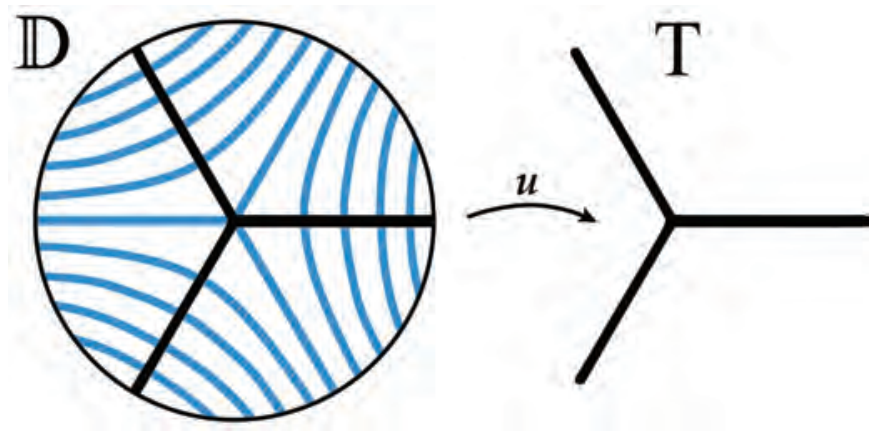

Figure 43. The energy-minimizing map $u$ takes each leaf (drawn in blue) to its unique point in the tripod $T$.

The regularity theorem of Gromov-Schoen asserts similar behavior for harmonic maps into Euclidean buildings.

Theorem 12. The singular set of an energy-minimizing map $u: \Omega \rightarrow X$ from a Lipschitz Riemannian domain into a Euclidean building is of Hausdorff codimension at least 2.

This is a delicate result and depends heavily on the special structure of the target space. We now give an example from Gromov-Schoen [GS] of a harmonic map with singular set of codimension $<2$. Unlike 2-dimensional Euclidean buildings, the 2-dimensional target space in this example has no isometric totally geodesic embedding of $\mathbb{R}^{2}$. 
Example 13. As in Figure 44 , let $\Omega=\mathbb{D}$ be the standard disk and let $X=\operatorname{Cone}(\Gamma)$ be the metric cone over a curve $\Gamma$ of length $2 \pi \alpha$ with vertex denoted by $P_{0}$. If $\alpha>1$, there exist closed nondegenerate and disjoint intervals $I_{1}, I_{2} \subset$ $\Gamma$ such that the length of each component of $\Gamma \backslash\left(I_{1} \cup I_{2}\right)$ is at least $\pi$. If $C\left(I_{i}\right)$ denotes the convex hull of $I_{i} \cup P_{0}$ in Cone $(\Gamma)$, then $C\left(I_{i}\right)$ is a sector and $K=C\left(I_{1}\right) \cup C\left(I_{2}\right)$ is the convex hull of $I_{1} \cup I_{2}$ in Cone $(\Gamma)$. Let

$$
u: \overline{\mathbb{D}} \rightarrow \text { Cone }(\Gamma)
$$

be the energy-minimizing map such that

$$
\left.u\right|_{\partial \mathbb{D}}: \partial \mathbb{D} \rightarrow \partial K=\partial C\left(I_{1}\right) \cup \partial C\left(I_{2}\right)
$$

is a constant-speed parameterization of the join of the closed curves $\partial C\left(I_{1}\right)$ and $\partial C\left(I_{2}\right)$. Observe that $u(\overline{\mathbb{D}}) \subset K$ since $K$ is geodesically convex and that $\overline{\mathbb{D}} \backslash u^{-1}\left(P_{0}\right)$ is not connected since $K \backslash\left\{P_{0}\right\}$ is not connected. Since $u^{-1}\left(P_{0}\right)=$ $S(u)$, we conclude that

$$
\operatorname{dim}_{\mathcal{H}}(S(u))=\operatorname{dim}_{\mathcal{H}}\left(u^{-1}\left(P_{0}\right)\right) \geq 1 .
$$

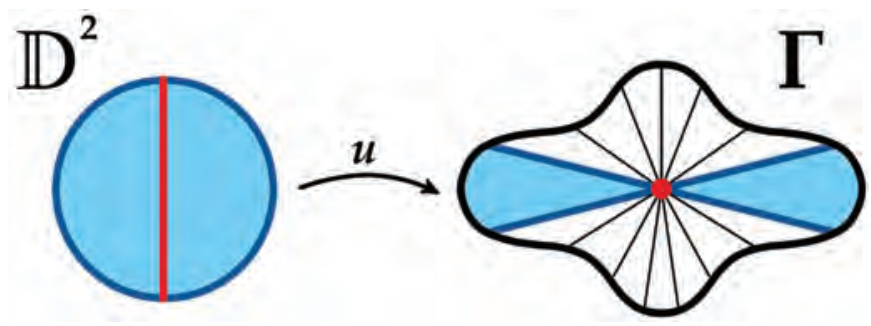

Figure 44. The singular set $S(u)$ (depicted in red on the left) of the energy-minimizing map $u$ from a disk, $\mathbb{D}^{2}$, into a cone, Cone $(\Gamma)$, over a curve, $\Gamma$, of length greater than $2 \pi$, with prescribed boundary (in dark blue), has Hausdorff dimension at least 1.

The key ingredient in the proof of Theorem 12 is the monotonicity property of energy-minimizing maps. This relies on the monotonicity property from the SchoenUhlenbeck theory combined with the convexity of the distance function in an NPC space $X$. Using monotonicity, Gromov-Schoen [GS] construct homogeneous maps approximating energy-minimizing maps similar to the tangent maps in the Schoen-Uhlenbeck theory. Furthermore, they introduce the notion of a homogeneous degree-1 map $l: \Omega \rightarrow X_{0}$ being effectively contained in an essentially regular totally geodesic subspace $X_{0}$. The metric space $X_{0}$ is called essentially regular if any harmonic map into $X_{0}$ is well approximated near a point by a homogeneous degree-1 map. The map $l$ being effectively contained in $X_{0}$ says that most points in the image of $l$ are sufficiently far away from $X \backslash X_{0}$. For example, the union of two copies of $[0, \infty)$ in $T$ (which we will call $L$ ) is essentially regular (since it can be isometrically identified to $\mathbb{R}$ ), and the identity map $l: \mathbb{R} \rightarrow L \approx \mathbb{R}$ is effectively contained in $L \subset T$.

Theorem 12 is derived from the following:
Theorem 14. Let $X_{0}$ be an essentially regular totally geodesic subspace of $X$ and let $l: \Omega \rightarrow X_{0}$ be a homogeneous degree 1 map effectively contained in $X_{0}$ with $l\left(x_{0}\right)=P_{0} \in$ $X_{0}$. If an energy-minimizing map $u: \Omega \rightarrow X$ is sufficiently close tol in $B_{r_{0}}\left(x_{0}\right)$, then $u\left(B_{\sigma_{0}}\left(x_{0}\right)\right) \subset X_{0}$ for some $\sigma_{0}>0$.

The proof of Theorem 14 [GS] is an analytical tour de force. It is reminiscent of the proof of the SchoenUhlenbeck $\epsilon$-regularity theorem, but it additionally overcomes the difficulty that there is no PDE to work with in the singular setting. The idea is to inductively compare successive blow-up scalings of $u$ with the energy-minimizing maps into $X_{0}$. The delicate argument combines the convexity property of the distance function in an NPC space with the properties of $X_{0}$ and $l$.

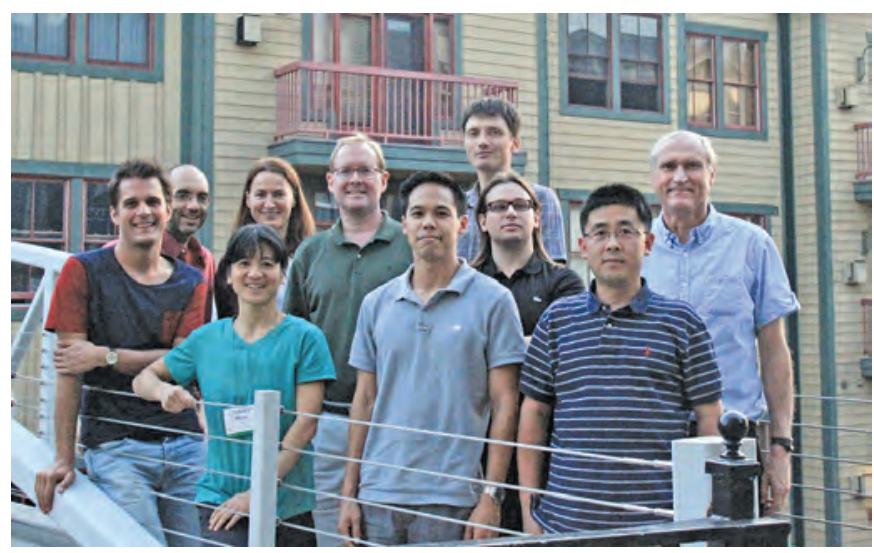

Figure 45. Schoen (far right) with some of his PhD students at Park City in 2013 at the 23rd Annual PCMI Summer Session. First row: Chikako Mese, Dan Lee, Xiaodong Wang. Second row: Michael Eichmair, Justin Corvino, Ailana Fraser, Hugh Bray, Alessandro Carlotto, Rick Schoen. Third row: Peter Topping (actually a student of Mario Micallef, Schoen's first student).

Rick's innovations opened up a completely new avenue in the applications of harmonic map theory. In particular, in a joint work of the author with Georgios Daskalopoulos, Rick's ideas were pushed forward to approach rigidity problems that could not be previously solved by other methods: superrigidity of hyperbolic buildings and holomorphic rigidity of Teichmüller space.

Daskalopoulos and Mese [DM1] extended the GromovSchoen regularity theory to include important NPC spaces other than Euclidean buildings. This result implies, as originally conjectured by Gromov, the superrigidity of hyperbolic buildings. Another application concerns the Weil-Petersson completion $\overline{\mathcal{T}}$ of Teichmüller space $\mathcal{T}$. The analysis of harmonic maps into $\overline{\mathcal{T}}$ is difficult because the Weil-Petersson metric is degenerate near the boundary.

Inspired by Rick's proof of Theorem 14, in a series of papers that culminated in [DM2], we introduced new techniques and proved the long-standing holomorphic rigidity conjecture of Teichmüller space. Loosely speaking, we proved that the action of the mapping class group 
uniquely determines the Teichmüller space as a complex manifold. The main ingredient in the proof is the surprising discovery that the Weil-Petersson completion $\overline{\mathcal{T}}$ of Teichmüller space contains essentially regular subspaces in a sense similar to Euclidean buildings despite its nonlocal compactness and degenerating geometry near the boundary.

\section{On a Personal Note}

Rick was a terrific thesis advisor for me at Stanford, and I have many fond memories of my time as a graduate student. I am grateful for his generosity, vast mathematical knowledge, and many words of wisdom that he has shared with me. Rick's powerful ideas have guided me throughout my career as a mathematician.

\section{References}

[DM1] G. DASKAlopoulos and C. MESE, On the singular set of harmonic maps into DM-complexes, Mem. Amer. Math. Soc. 239 (2016), no. 1129, v+89 pp. MR3431944

[DM2] G. DASKAlopoulos and C. Mese, Rigidity of Teichmüller space, preprint.

[GS] M. GRomov and R. Schoen, Harmonic maps into singular spaces and $p$-adic superrigidity for lattices in groups of rank one, IHES Publ. Math. 76 (1992), 165-246. MR1215595

[KS1] N. KorevaAR and R. Schoen, Sobolev spaces and harmonic maps into metric space targets, Comm. Anal. Geom. 1 (1993), 561-659. MR1266480

[KS2] N. KOREVAAR and R. SchOEN, Global existence theorems for harmonic maps to non-locally compact spaces, Comm. Anal. Geom. 5 (1997), 333-387. MR1483983

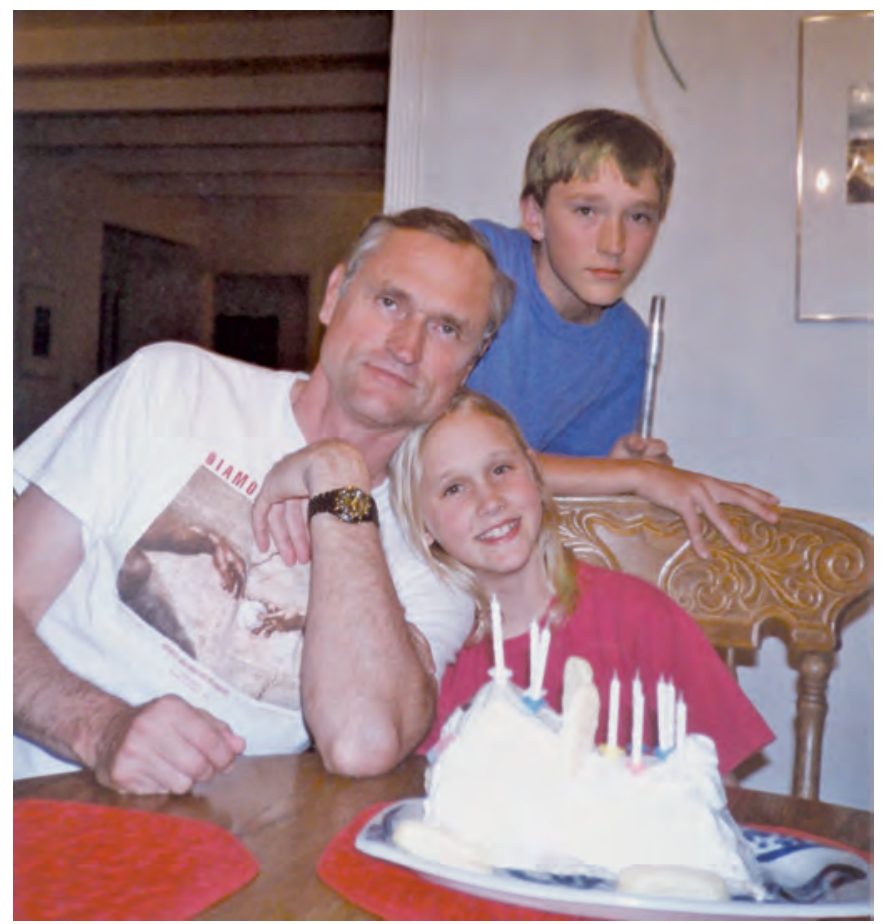

Figure 46. Rick Schoen with he and wife Doris Fischer-Colbrie's two children Alan and Lucy.

\section{Ailana Fraser}

\section{Steklov Eigenvalues and Free Boundary Minimal Surfaces}

One of the fundamental problems in spectral geometry is to determine sharp eigenvalue bounds. This is a subject with a long tradition and is a classical subject both in geometry and PDE. Work for compact surfaces extends over a period of more than forty years, with contributions by many authors. Determining sharp eigenvalue bounds is related to finding extremal metrics for the eigenvalue problem. The existence of extremal metrics has been a very difficult subject, and Richard Schoen has contributed in a fundamental way.

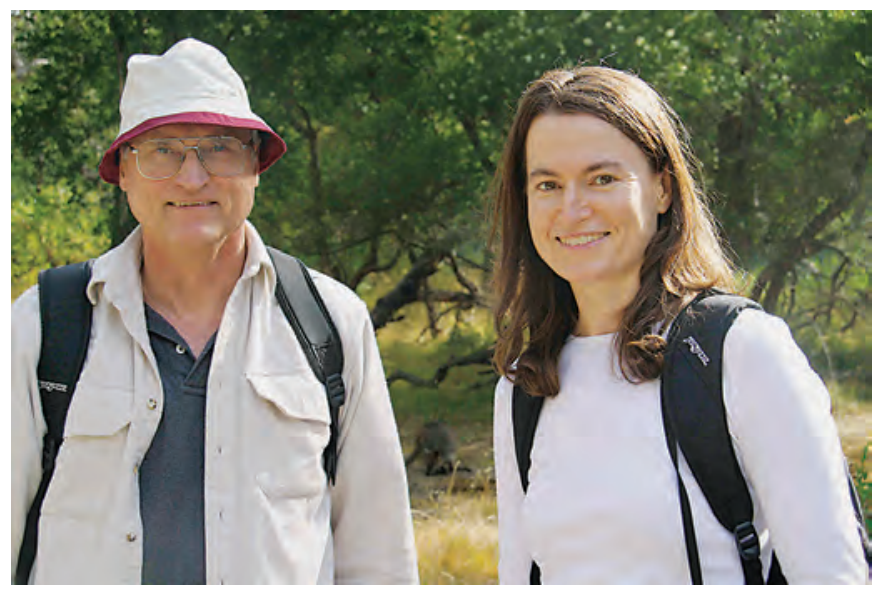

Figure 47. R. Schoen and A. Fraser, Workshop on General Relativity and Geometric Analysis, Australia, 2010. Can you find the kangaroo?

For surfaces $M$ with boundary, the eigenvalue problem that leads to a geometrically interesting variational problem is the Steklov eigenvalue problem:

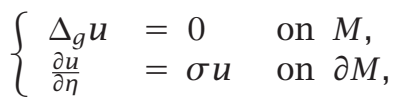

where $g$ is a Riemannian metric on $M, \eta$ is the outward unit normal vector to $\partial M, \sigma \in \mathbb{R}$, and $u \in C^{\infty}(M)$.

Steklov eigenvalues are eigenvalues of the Dirichlet-toNeumann map, $\Lambda$, which takes a given smooth function, $f: \partial M \rightarrow \mathbb{R}$, finds its harmonic extension, $u: M \rightarrow \mathbb{R}$, satisfying

$$
\begin{array}{cc}
\Delta u=0 & \text { on } M, \\
u=f & \text { on } \partial M,
\end{array}
$$

and gives as an output the normal derivative of that harmonic extension,

$$
\Lambda(f)=\frac{\partial u}{\partial \eta}
$$

Ailana Fraser is professor of mathematics at the University of British Columbia. Her email address is afraser@math.ubc.ca. 


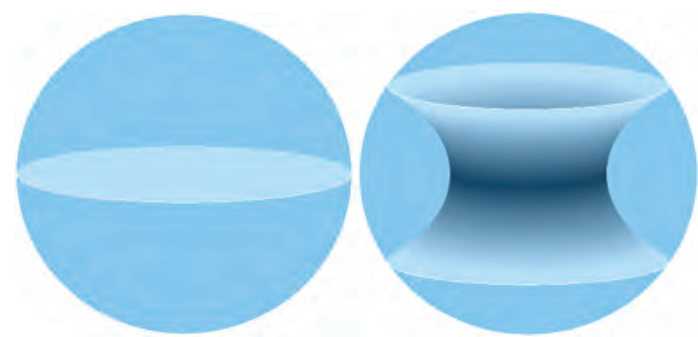

Figure 48. Examples of free boundary minimal surfaces in the ball include the equatorial plane and the critical catenoid.

The Dirichlet-to-Neumann map is a self-adjoint elliptic pseudo-differential operator of order 1 and therefore has discrete spectrum

$$
\sigma_{0}=0<\sigma_{1} \leq \sigma_{2} \leq \cdots \leq \sigma_{k} \leq \cdots \rightarrow \infty .
$$

The Steklov problem has a long history and many people have contributed; please see Girouard and Polterovich [GP] for a recent survey.

Some basic questions are:

1) Assuming we fix the boundary length to be 1, what is the metric on $M$ that maximizes the first eigenvalue?

2) Does such a metric exist?

3) If so, what can we say about its geometry?

If we fix a surface $M$ of genus $\gamma$ with $k$ boundary components, define

$$
\sigma^{*}(\gamma, k)=\sup _{g} \sigma_{1}(g) L_{g}(\partial M),
$$

where the supremum is over all smooth metrics on $M$. By a 1954 result of Weinstock, $\sigma^{*}(0,1)=2 \pi$, and the supremum is achieved by the Euclidean disk. In general, there is a coarse upper bound due to Fraser-Schoen [FS1] and Kokarev:

$$
\sigma^{*}(\gamma, k) \leq \min \{2 \pi(\gamma+k), 8 \pi[(\gamma+3) / 2]\} .
$$

It turns out that there is an intimate connection between maximizing metrics and minimal surfaces in the Euclidean unit ball $B^{n}$ that are proper in the ball and that meet the boundary of the ball orthogonally. Such surfaces are referred to as free boundary minimal surfaces since they arise variationally as critical points of the area among surfaces in the ball whose boundaries lie on $\partial B^{n}$ but are free to vary on $\partial B^{n}$. Classical examples (see Figure 48) include the equatorial plane disk and the critical catenoid, the unique portion of a suitably scaled catenoid that defines a free boundary surface in $B^{3}$.

Free boundary minimal surfaces $\Sigma$ in $B^{n}$ are characterized by the condition that the coordinate functions are Steklov eigenfunctions with eigenvalue 1:

$$
\begin{array}{cc}
\Delta x_{i}=0 & \text { on } \Sigma, \\
\nabla_{\eta} x_{i}=x_{i} & \text { on } \partial \Sigma .
\end{array}
$$

Moreover, if we assume that we have a smooth metric $g$ that realizes the maximum, then there are independent first eigenfunctions $u_{1}, \ldots, u_{n}$ such that the map

$$
u=\left(u_{1}, \ldots, u_{n}\right)
$$

defines a proper conformal map from $M$ into $B^{n}, n \geq 3$. The image $\Sigma=u(M)$ is a free boundary minimal surface in $B^{n}$, and the maximizing metric can be realized by the induced metric [FS2].

The question of existence of a maximizing metric is extremely difficult: a major achievement of our work [FS2] is the proof that for any compact surface $M$ of genus zero with boundary, a smooth maximizing metric $g$ exists. More generally, existence of a maximizing metric on any surface $M$ with boundary is proved, provided the conformal structure is controlled for any metric near the maximum. The proof involves a canonical regularization procedure that produces a special maximizing sequence for which a carefully chosen set of eigenfunctions converges strongly in $H^{1}$ to a limit. It is then shown that the limit defines a continuous map that is stationary for the free boundary problem. Higher regularity follows from minimal surface theory.

For surfaces of genus zero with arbitrarily many boundary components, we prove boundedness of the conformal structure for nearly maximizing metrics and hence existence of a maximizing metric:

Theorem 15. For any $k \geq 1$ there exists a smooth metric $g$ on the surface of genus 0 with $k$ boundary components with the property $\sigma_{1}(g) L_{g}(\partial M)=\sigma^{*}(0, k)$.

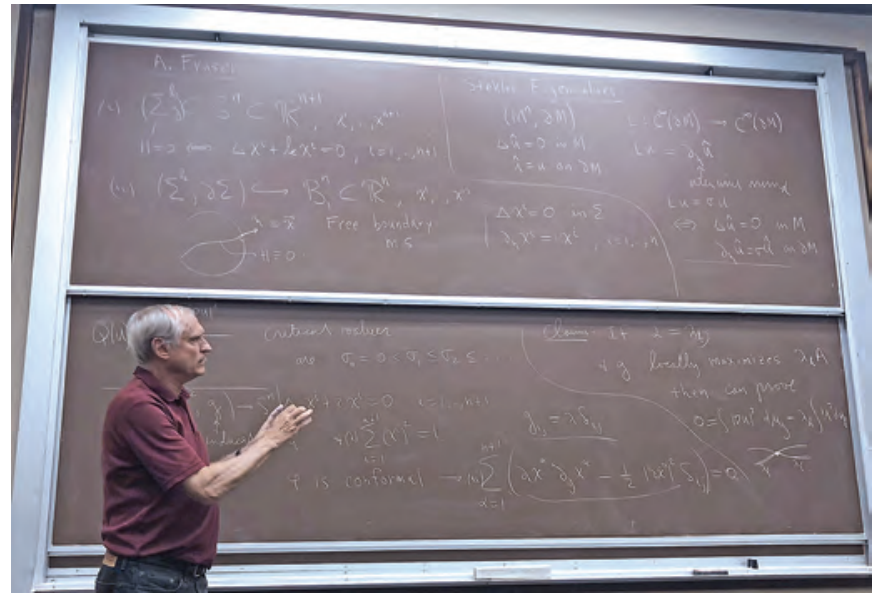

Figure 49. Schoen presenting his work with Fraser at Stanford in 2018.

In the case of the annulus and the Möbius band, we explicitly characterize the maximizing metric. This follows from a characterization of the critical catenoid and the "critical Möbius band," an explicit free boundary minimal embedding of the Möbius band into $B^{4}$ by first eigenfunctions, as the only free boundary minimal immersions of the annulus and Möbius band into $B^{n}$ by first eigenfunctions.

As a result we have sharp eigenvalue bounds:

Theorem 16. For any metric annulus $M$,

$$
\sigma_{1} L \leq\left(\sigma_{1} L\right)_{c c},
$$

with equality if and only if $M$ is equivalent to the critical catenoid. In particular,

$$
\sigma^{*}(0,2)=\left(\sigma_{1} L\right)_{c c} \approx 4 \pi / 1.2 .
$$




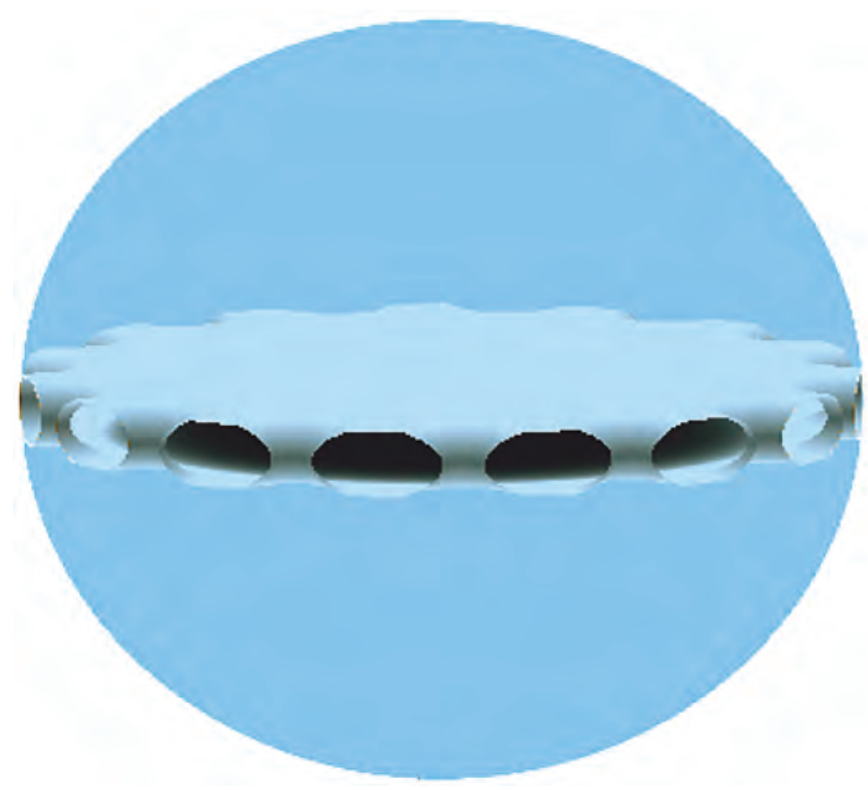

Figure 50. Free boundary minimal surface $\Sigma_{k}$ of genus 0 with $k$ boundary components in $B^{3}$ as $k \rightarrow \infty$. For large $k, \Sigma_{k}$ is approximately a pair of nearby parallel plane disks joined by $k$ half-catenoidal boundary bridges.

Theorem 17. For any metric Möbius band $M$,

$$
\sigma_{1} L \leq\left(\sigma_{1} L\right)_{c m b}=2 \pi \sqrt{3},
$$

with equality if and only if $M$ is equivalent to the critical Möbius band.

For surfaces of genus 0 with $k \geq 3$ boundary components, while we don't have an explicit characterization of the maximizing metrics, we [FS2] show that maximizing metrics arise from free boundary surfaces in $B^{3}$ which are embedded and star-shaped with respect to the origin. We analyze the limit as the number of boundary components tends to infinity, as in Figure 50, and obtain an asymptotically sharp eigenvalue bound:

Theorem 18 ([FS2, Fraser and Schoen]). The sequence $\sigma^{*}(0, k)$ is strictly increasing in $k$ and

$$
\lim _{k \rightarrow \infty} \sigma^{*}(0, k)=4 \pi \text {. }
$$

For each $k$ a maximizing metric is achieved by a free boundary minimal surface $\Sigma_{k}$ in $B^{3}$ of area less than $2 \pi$. The limit of these minimal surfaces as $k$ tends to infinity is a double disk. See Figure 50.

The only previously known free boundary minimal surfaces in $B^{3}$ were the equatorial disk and the critical catenoid. As a consequence of Theorem 18, we have the following existence theorem for free boundary minimal surfaces in the ball.

Corollary 19. For every $k \geq 1$ there is an embedded free boundary minimal surface in $B^{3}$ of genus 0 with $k$ boundary components. Moreover these surfaces are embedded by first eigenfunctions.
Recently Folha-Pacard-Zolotareva, Kapouleas-Li, and Kapouleas-Wiygul have used gluing techniques and Ketover has used an equivariant min-max construction to prove existence of further new examples of free boundary minimal surfaces in $B^{3}$.

\section{On a Personal Note}

I was a PhD student of Richard Schoen's at Stanford University from 1993 to 1998. One could not hope for a better advisor, and I will feel forever fortunate and grateful to have had the opportunity to work with him. Not only is the mathematics exciting, but another aspect that is so special about working with him is how motivating and inspiring he is in discussions and lectures. It has been a privilege to collaborate with him many years later.

\section{References}

[FS1] A. FrASER and R. SCHOEN, The first Steklov eigenvalue, conformal geometry, and minimal surfaces, Adv. Math. 226 (2011), no. 5, 4011-4030. MR2770439

[FS2] A. FrASER and R. SCHOEN, Sharp eigenvalue bounds and minimal surfaces in the ball, Invent. Math. 203 (2016), no. 3, 823-890. MR3461367

[GP] A. GirouARD and I. POLTERovich, Spectral geometry of the Steklov problem, J. Spectral Theory 7 (2017), 321-359. MR3662010

\section{Image Credits}

Figure 1 courtesy of the Schoen family.

Figures 2 and 25 courtesy of Richard Schoen.

Figure 3 courtesy of Robert Kusner, photo editing by Pen Chang. Figure 4 by Christina Sormani, with photo editing by Pen Chang. Figures 5, 20, 35, and 49 by Christina Sormani.

Figures $6,12,13,16,45$, and 47 courtesy of Michael Eichmair. Figures 7, 9, 10, 17, 37, and 40-44 by Pen Chang.

Figure 8 by Robert Kusner.

Figure 11 by Damin Wu.

Figure 14 courtesy of Lixin Qin.

Figures 15 and 46 courtesy of Doris Fischer-Colbrie.

Figure 18 by Christina Sormani, with photo editing by Pen Chang, including virtual refilling of Minicozzi's glass.

Figure 19 by Christina Sormani of soap film by Julinda Pillati.

Figure 21 by Kuo-Wei Lee.

Figures 22 and 24 photographer unknown. From the Shelby White and Leon Levy Archives Center, Institute for Advanced Study, Princeton, NJ, USA.

Figure 23 courtesy of Chuulian Terng.

Figures 26, 27, and 33 by Matthias Weber; see www. indiana. edu / minimal/archive/. Licensed under a Creative Commons Attribution-Noncommercial-No Derivative Works 3.0 Unported License.

Part of Figures 28-31 enhanced photos by Christina Sormani of films by Kendall Chang and Tabitha Chang.

Figure 32 by Nick Schmitt, courtesy of GANG (www.gang.umass . edu).

Figure 33 by Matthias Weber see www. indiana.edu/ minimal /archive/. Licensed under a Creative Commons AttributionNoncommercial-No Derivative Works 3.0 Unported License.

Figure 34 courtesy of John Sullivan.

Figures 36 and 39 courtesy of Fernando Codá Marques. 
Figure 38 by Severino Marques.

Figures 48 and 50 by Emma Fajeau.

Photo of Hugh Bray courtesy of Hugh Bray.

Photo of Fernando Codá Marques courtesy of Fernando Codá Marques.

Photo of Michael Eichmair courtesy of Michael Eichmair.

Photo of Ailana Fraser courtesy of Ailana Fraser.

Photo of Lan-Hsuan Huang by Damin Wu.

Photo of Robert Kusner courtesy of Robert Kusner.

Photo of Chikako Mese by Will Kirk.

Photo of William P. Minicozzi II by Bryce Vickmark/courtesy of MIT.

Photo of Karen Uhlenbeck courtesy of Chuulian Terng.

Photo of Shing-Tung Yau courtesy of Michael Eichmair.

\section{ABOUT THE AUTHORS}

Hugh Bray completed his doctorate with Schoen in 1997. He is an American Mathematical Society Fellow and was a Sloan Research Fellow.

Fernando Codá Marques was a student of José Escobar, who was a student of Schoen. He was awarded the ICTP Ramanujan Prize in 2012 and the Oswald Veblen Prize in Geometry with André Neves in 2016.

Michael Eichmair completed his doctorate with Schoen in 2008. In 2016 he won the Austrian START-Preis for young scientists.

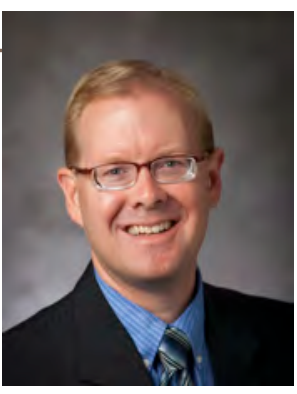

Hugh Bray

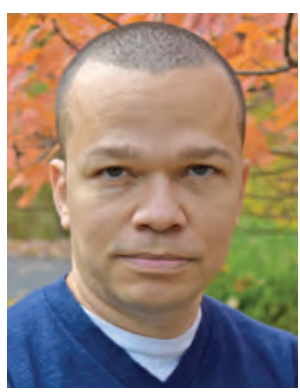

Fernando Codá Marques

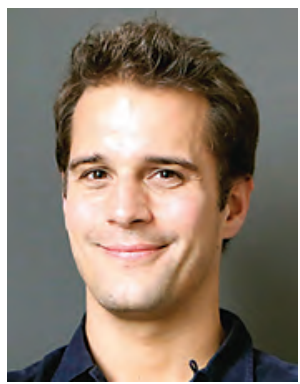

Michael Eichmair
Ailana Fraser completed her doctorate with Schoen in 1998. She won the Canadian Krieger-Nelson Prize and is a Fellow of the American Mathematical Society.

Lan-Hsuan Huang completed her doctorate with Schoen in 2009. She was awarded an NSF Career Grant in 2015. She is a Simons Fellow and a von Neumann Fellow.

Robert Kusner completed his doctorate with Schoen in 1988. He has led the Center for Geometry, Analysis, Numerics and Graphics (GANG) and was most recently a visiting fellow at ICERM.

Chikako Mese completed her doctorate with Schoen in 2006. She has been a Simons Fellow and has NSF funding for her research on harmonic maps.

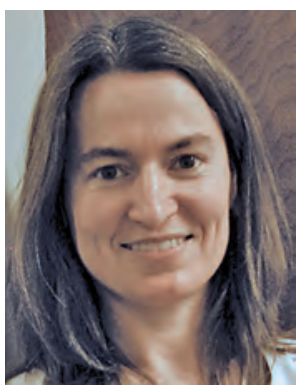

Ailana Fraser

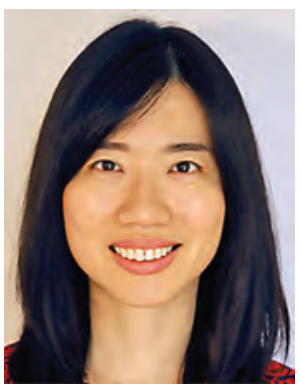

Lan-Hsuan Huang

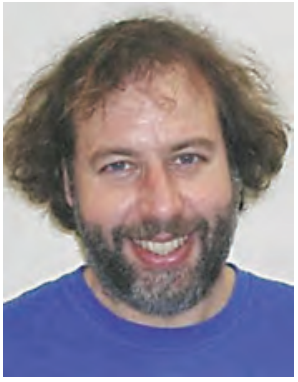

Robert Kusner

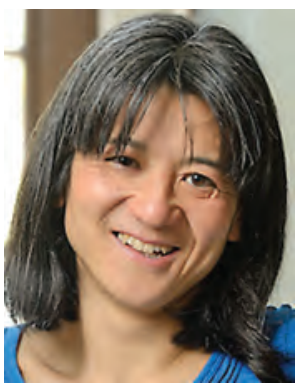

Chikako Mese 


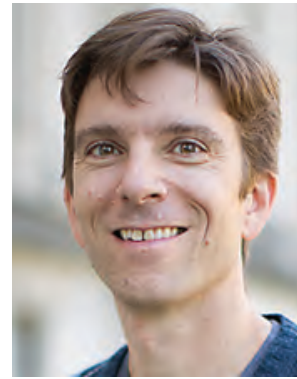

William

P. Minicozzi II

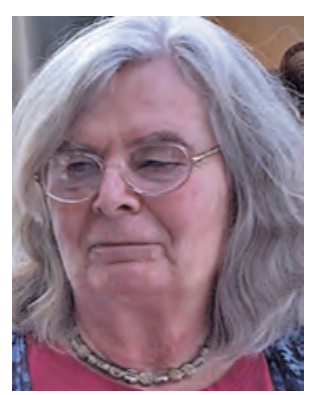

Karen Uhlenbeck

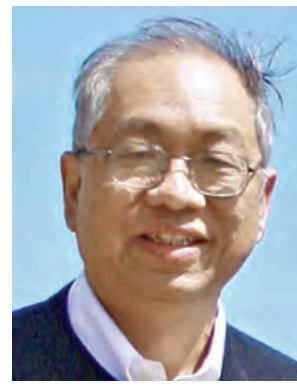

Shing-Tung Yau

William P. Minicozzi II completed his doctorate with Schoen in 1994. In 2010 he and Tobias Colding were awarded the Oswald Veblen Prize in Geometry for their work on minimal surfaces.

Karen Uhlenbeck is a MacArthur Fellow, Fellow of AAAS, and AMS Fellow. She won the National Medal of Science in 2000 and the AMS Steele Prize in 2007 "for her foundational contributions in analytic aspects of mathematical gauge theory."

Shing-Tung Yau has won the Fields Medal for "his contributions to partial differential equations, to the Calabi conjecture in algebraic geometry, to the positive mass conjecture of general relativity theory, and to real and complex MongeAmpère equations." In 1997 he was awarded the US National Medal of Science. In 2010 he won the Wolf Prize in Mathematics. 